PRE, submitted

November 4, 2018

\title{
Analytic Calculation of the Anomalous Exponents in Turbulence: Using the Fusion Rules to Flush Out a Small Parameter
}

\author{
Victor S. L'vov and Itamar Procaccia \\ Department of Chemical Physics, The Weizmann Institute of Science, Rehovot 76100, Israel
}

\begin{abstract}
The main difficulty of statistical theories of fluid turbulence is the lack of an obvious small parameter. In this paper we show that the formerly established fusion rules can be employed to develop a theory in which Kolmogorov's statistics of 1941 acts as the zero order, or background statistics, and the anomalous corrections to the K41 scaling exponents $\zeta_{n}$ of the $n$th order structure functions can be computed analytically. The crux of the method consists of renormalizing a 4point interaction amplitude on the basis of the fusion rules. The novelty is that this amplitude includes a small dimensionless parameter, which is shown to be of the order of the anomaly of $\zeta_{2}, \delta_{2}=\zeta_{2}-2 / 3 \approx 0.03$. Higher order interaction amplitudes are shown to be even smaller. The corrections to K41 to $O\left(\delta_{2}\right)$ result from standard logarithmically divergent ladder-diagrams in which the 4-point interaction acts as a "rung". The theory allows a calculation of the anomalous exponents $\zeta_{n}$ in powers of the small parameter $\delta_{2}$. The $n$-dependence of the scaling exponents $\zeta_{n}$ stems from pure combinatorics of the ladder diagrams. In this paper we calculate the exponents $\zeta_{n}$ up to $O\left(\delta_{2}^{3}\right)$. Previously derived bridge relations allow a calculation of the anomalous exponents of correlations of the dissipation field and of dynamical correlations in terms of the same parameter $\delta_{2}$. The actual evaluation of the small parameter $\delta_{2}$ from first principles requires additional developments that are outside the scope of this paper.
\end{abstract}

\section{INTRODUCTION}

The aim of this paper is to build on previous work to achieve a controlled evaluation of the anomalous exponents that characterize various correlation and structure function in Navier-Stokes turbulence, and in particular the exponents $\zeta_{n}$ that characterize $n$th order structure functions. The main result of this paper is that given a single experimental input (for example the value of the anomalous exponent of the second order structure function), the $n$ dependence of all the other exponents that were reliably measured in experiments and simulations can be calculated analytically.

Decades of experimental and theoretical attention (see for example [1 [ 6]) have been devoted to two types of simultaneous correlation functions; the first type includes the structure functions of velocity differences,

$$
S_{n}(\boldsymbol{R})=\left\langle|\boldsymbol{u}(\boldsymbol{r}+\boldsymbol{R})-\boldsymbol{u}(\boldsymbol{r})|^{n}\right\rangle,
$$

where $\langle\ldots\rangle$ stands for a suitably defined ensemble average. A second type of correlations include gradients of the velocity field. An important example is the rate $\epsilon(\boldsymbol{r}, t)$ at which energy is dissipated into heat due to viscous damping. This rate is roughly $\nu|\nabla \boldsymbol{u}(\boldsymbol{r}, t)|^{2}$. An often-studied simultaneous correlation function of $\hat{\epsilon}(\boldsymbol{r}, t)=\epsilon(\boldsymbol{r}, t)-\bar{\epsilon}$ is

$$
K_{\epsilon \epsilon}(\boldsymbol{R})=\langle\hat{\epsilon}(\boldsymbol{r}+\boldsymbol{R}) \hat{\epsilon}(\boldsymbol{r})\rangle .
$$

It has been hypothesized by Kolmogorov in 1941 (K41) and 1962 (K62) that statistical objects of this type exhibit power law dependence on $R$ [1],7]:

$$
S_{n}(R) \propto R^{\zeta_{n}}, \quad K_{\epsilon \epsilon}(R) \propto R^{-\mu} .
$$

In addition, the K41 theory predicted the values of $\zeta_{n}$ to be $n / 3$. Experimental measurements and computer simulations show that in some aspects K41 was remarkably close to the truth. The major aspect of its predictions, that the statistical quantities depend on the length scale $R$ as power laws, is corroborated by experiments. On the other hand, the predicted exponents seem not to be exactly realized. The numerical values of $\zeta_{n}$ deviate progressively from $n / 3$ when $n$ increases [3,5]. K62 tried to improve on this prediction by taking into account the fluctuations in the rate of energy dissipation. On the basis of a phenomenological model, assuming the distributions function of energy dissipation to be lognormal, K62 reached the predictions

$$
\zeta_{n}=\frac{n}{3}-\frac{\mu n(n-3)}{18} .
$$

Besides the fact that these predictions did not follow from fluid mechanical considerations, it was pointed out [6] that they are violating basic inequalities that do not allow the exponents $\zeta_{n}$ to decrease, something that always happens with (A) with $n$ large enough. The quest for computing the scaling exponents from the equations of fluid mechanics was long, arduous, and on the whole pretty unsuccessful.

In this paper we present an approach that is based on our own previous findings which culminates in the analytic calculation of exponents like $\zeta_{n}$ and $\mu$. At present 
the calculation is not completely from first principle. We need the input of one number from experiment, say $\delta_{2} \equiv \zeta_{2}-2 / 3$. Given this number we can calculate all the other exponents systematically with $\delta_{2}$ being a small parameter that organizes our calculations. To first order in $\delta_{2}$ we recapture (4). We will show that the result to $O\left(\delta_{2}\right)$ is universal, independent of the details of the calculations performed below. To second order we find the results

$$
\begin{aligned}
\zeta_{n} & =\frac{n}{3}-\frac{n(n-3)}{2} \delta_{2}\left[1+2 \delta_{2}(n-2) b_{2}\right]+O\left(\delta_{2}^{3}\right), \\
\mu & =9 \delta_{2}\left(1+8 b_{2} \delta_{2}\right)+O\left(\delta_{2}^{3}\right) .
\end{aligned}
$$

The curves of $\zeta_{n}$ vs. $n$ are shown in Fig. 11. We show the K41 prediction, the result of our calculation to 1-loop order, and the 2-loop result that is presented in Eq. (5). While the form of these results is universal, the numerical value of the dimensionless parameter $b_{2}$ depends on the details of the calculations; we find that $b_{2}$ is always negative and of the order of unity. Note that the 2-loop results correct the unwanted down curving of the 1-loop calculation (which is the same malaise as in K62).

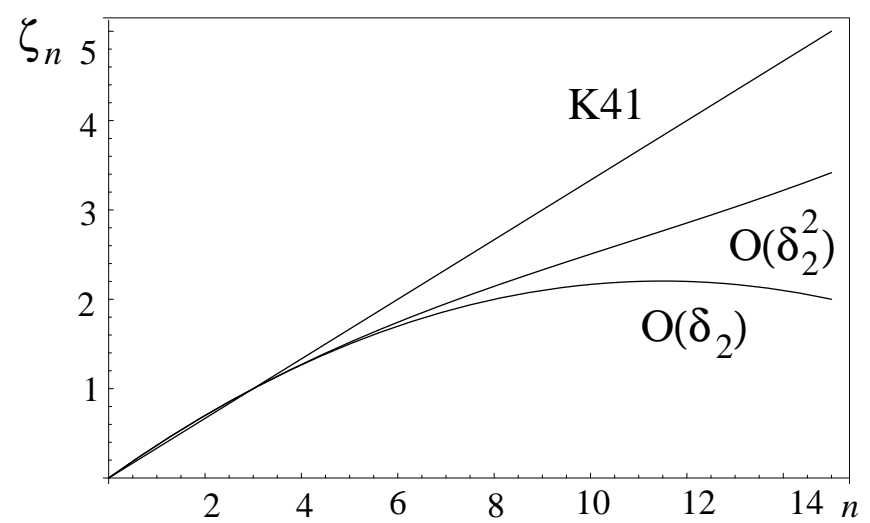

FIG. 1. The scaling exponents $\zeta_{n}$ as a function of $n$. The calculation is organized by the small parameter $\delta_{2}=\zeta_{2}-2 / 3 \approx 0.03$. Shown is the K41 prediction which is zero-order in $\delta_{2}$, together with our results to first and second order in $\delta_{2}$. To first order the results are the same as the phenomenological prediction of K62, and to 2nd order it is Eq. (5) with $b_{2}=-0.55$ according to Eq. (72).

In thinking about the strategy for this work we were led by some insights that developed in the context of understanding how to compute the scaling exponents of the Kraichnan model of passive scalar advection 8.9. In that model a scalar field $T(\boldsymbol{r}, t)$ is advected by a Gaussian velocity field $\boldsymbol{u}(\boldsymbol{r}, t)$ which is delta-correlated in time but which has a scaling exponent $\zeta_{2}=\epsilon$. For $\epsilon=0$ the advected scalar has trivial statistics, and for $\epsilon$ small the model has a natural small parameter. It turned out that the calculation of the exponents can proceed along two ways. The first, which is non-perturbative, was pioneered in [10]. It considers the differential equations that the $n$th order correlation functions satisfy, and identifies the anomalous scaling solutions as the zero modes of these differential equations. The calculation of the exponents then depends on the calculation of the zero modes themselves, a task that is not at all easy, and therefore such calculations were never done for any order but $O(\epsilon)$. In this method the renormalization scale is the outer scale $L$, not the inner scale $\eta$, and the dimensionless ratio of scales that carries the anomalous part of the exponents of the structure functions is $L / R$ where $R$ is defined in Eq. (1). A second method that was discussed in detail in [11] considers instead of the correlation functions the averages of higher moments of $\nabla T$, i.e. $\left\langle|\nabla T|^{n}\right\rangle$. These quantities diverge as powers of $L / \eta$, and the exponents of this power are the same as the anomalous part of the exponent of the $n$th order structure function. The great advantage of the second method is that one can write a perturbative theory in $\epsilon$ for the scaling exponents themselves, without any need to compute the zero modes or any other functions of many variables. Thus the second method allows easily computations to $O\left(\epsilon^{2}\right)$ 12, 13 and with some more efforts to higher orders. The insight gained is that one needs to focus on a quantity that offers the most feasible calculation, exposing the anomalous part of the exponents as it appears with a dimensionless ratio of length scales.

In Navier Stokes turbulence the situation is similar. On the one hand we have a non-perturbative theory which in this case is the infinite hierarchy formed by the equations of motion of the correlation function [14]. We can use this hierarchy to demonstrate that anomalous solutions exist, but the computation of the scaling exponents requires a calculation of the correlation functions themselves. This is a very difficult task that up to now was not accomplished in a satisfactory manner. The other approach will be described in this paper. It will be a perturbative theory for the scaling exponents themselves, not requiring the computation of the correlation function along the way. Similarly to the second method in the Kraichnan problem it will be based on considering limits of correlation functions when $p$ coordinates fuse. In that limit we create, even when all the distances are in the inertial range, a ratio of large and small lengthscales that appears raised to the power of the anomalous exponent $\zeta_{n}$.

The two previous findings that influence crucially the present formulation are the mechanism for anomalous scaling that was announced in Ref. [15], and the fusion rules that were discussed in Ref. [16]. In short, Ref. [15] exposed the ladder diagrams which appear in the theory of turbulence. These diagrams contain logarithmic divergences that are summable to power laws with anomalous exponents. These ladder diagrams contain "rungs" of the ladder, that are actually vertices with four, six and more "legs", representing 4-point, 6-point and higher order interaction amplitudes. In Ref. 15] these objects were rep- 
resented in terms of infinite series of diagrams that could not be resummed analytically. This is where the fusion rules are now called to save the day. The fusion rules determine the asymptotic properties of $n$-point correlation functions when subgroups of $p$ coordinates coalesce together. As such the fusion rules are non-perturbative, and are believed to be exact. We use the fusion rules to determine the asymptotic properties of the rungs. This is done such that a calculation of $\zeta_{2}$ from the theory will agree with the experimental value of $\zeta_{2}$. We then show that the knowledge of the asymptotics suffices for constructing a calculation of all the other scaling exponents, and in particular of $\zeta_{n}$ for $n>2$ and of $\mu$. The crux is that in the process of determining the analytic form of the rungs we discover that their amplitude contains powers of a dimensionless small parameter $\delta_{2}=\zeta_{2}-2 / 3 \approx 0.03$. Using this small parameter in the renormalized 4 -point interaction allows us to develop a systematic expansion in orders of $\delta_{2}$. At the end of this paper we sketch a way to understand the remaining task regarding the origin of the small parameter $\zeta_{2}-2 / 3$.

In Sect. II we summarize past results that are necessary for the present developments. In Sect. III we show how the fusion rules can be used to determine the properties of the rungs in the ladder diagrams appearing in the $n$th order correlation functions. The first important new result is demonstrated in Sect. V - the numerical coefficient contained in the 4-point rung is shown to be small, of the order of the anomaly of $\zeta_{2}$. This result is crucial since it allows (to our knowledge for the first time) to develop a perturbative calculation of the anomalous parts of all the other exponents. The physical reason for this result is that ( in Sect. IV) we are developing the theory around the K41 solution instead of the dissipative solution as was always attempted. In Sect. $\mathrm{V}$ we pave the way for the calculation of all the other exponents. In Sect. VI we calculate the scaling exponents by resumming the logarithmically divergent ladder diagrams up to $O\left(\delta_{2}\right)$ (which is known in the field-theoretic jargon as the "1-loop order" in the renormalized rungs). We find (admittedly to our surprise) that to this order the scaling exponents are identical to K62. Like the latter they suffer from the violation of the known requirement that $\zeta_{n}$ cannot decrease with $n$ [6]. In Sect. VII we show that the 2-loop order cures the malaise of K62, and we present the result (5) for $\zeta_{n}$ that in our theoretical estimate is valid for $n \leq 12$. The exponent $\mu$ is also computed in this Section. If one wanted results for $\zeta_{n}$ with higher values of $n$ one would need to go to 3-loop order (and see Sect. VIII where the form of $\zeta_{n}$ to this order is presented), but the present experimental situation does not warrant a theoretical prediction of $\zeta_{n}$ for very high values of $n$. In Sect. VIII we summarize the paper, paying special attention to the range of validity of the theory and to demonstrating that no uncontrolled approximations were made.

\section{SUMMARY OF PERTINENT PREVIOUS RESULTS}

In this Section we present a brief summary of some past work which is most pertinent. We refer to 18] as Paper I, to [15 as Paper II and to [17] as Paper III.

\section{A. The basic perturbation theory}

The starting point of the analysis are the Navier-Stokes equations for the velocity field of an incompressible fluid with kinematic viscosity $\nu$ which is forced by an external force $\boldsymbol{f}(\boldsymbol{r}, t)$ :

$$
\left(\frac{\partial}{\partial t}-\nu \Delta^{2}\right) \boldsymbol{u}+\stackrel{\leftrightarrow}{\boldsymbol{P}}(\boldsymbol{u} \cdot \nabla) \boldsymbol{u}=\stackrel{\leftrightarrow}{\boldsymbol{P}} \boldsymbol{f}
$$

where $\stackrel{\leftrightarrow}{\boldsymbol{P}}$ is the transverse projection operator $\stackrel{\leftrightarrow}{\boldsymbol{P}} \equiv$ $-\Delta^{-2} \boldsymbol{\nabla} \times \boldsymbol{\nabla} \times$. It is well known, (and see for example Paper I) that developing a perturbative approach 119 21 for the correlation functions and response functions in terms of the Eulerian velocity $\boldsymbol{u}(\boldsymbol{r}, t)$ results in a theory that is plagued with infra-red divergences. On the other hand one can transform to new variables, and after the transformation (which amounts to infinite partial resummations in the perturbation theory) one finds a renormalized perturbation theory that is finite, without any divergences in any order of the expansion (cf. 22 and Paper I) . One can achieve such a theory using Lagrangian variables 23]; we find it technically simpler to employ the Belinicher-L'vov transformation 22],

$$
\left.\boldsymbol{v}\left[\boldsymbol{r}_{0} \mid \boldsymbol{r}, t\right] \equiv \boldsymbol{u}\left[\boldsymbol{r}+\boldsymbol{\rho}\left(\boldsymbol{r}_{0}, t\right), t\right)\right]
$$

where $\boldsymbol{\rho}\left(\boldsymbol{r}_{0}, t\right)$ is the Lagrangian trajectory of a fluid point which has started at point $\boldsymbol{r}=\boldsymbol{r}_{0}$ at time $t=t_{0}$

$$
\boldsymbol{\rho}\left(\boldsymbol{r}_{0}, t\right)=\int_{0}^{t} \boldsymbol{u}\left[\boldsymbol{r}+\boldsymbol{\rho}\left(\boldsymbol{r}_{0}, \tau\right), \tau\right] d \tau
$$

The natural variables for a divergence free theory are the velocity differences

$$
\boldsymbol{w}\left(\boldsymbol{r}_{0} \mid \boldsymbol{r}, t\right) \equiv \boldsymbol{v}\left[\boldsymbol{r}_{0} \mid \boldsymbol{r}, t\right]-\boldsymbol{v}\left[\boldsymbol{r}_{0} \mid \boldsymbol{r}_{0}, t\right]
$$

Since the averages of quantities that depend on one time only can be computed at $t=0$, it follows that the average moments of these BL-variables are the structure functions of the Eulerian field $S_{n}(\boldsymbol{R})$ defined by Eq. (1). It was shown 22 that these variables satisfy the Navier Stokes equations, and that one can develop (cf. Paper I) a perturbation theory of the diagrammatic type in which the natural quantities are the Green's function $G_{\alpha \beta}\left(\boldsymbol{r}_{0} \mid \boldsymbol{r}, \boldsymbol{r}^{\prime}, t, t^{\prime}\right)$ and the correlation function $F_{\alpha \beta}\left(\boldsymbol{r}_{0} \mid \boldsymbol{r}, \boldsymbol{r}^{\prime}, t, t^{\prime}\right)$ : 


$$
\begin{aligned}
G_{\alpha \beta}\left(\boldsymbol{r}_{0} \mid \boldsymbol{r}, \boldsymbol{r}^{\prime}, t, t^{\prime}\right) & =\left.\frac{\delta\left\langle w_{\alpha}\left(\boldsymbol{r}_{0} \mid \boldsymbol{r}, t\right)\right\rangle}{\delta f_{\beta}\left(\boldsymbol{r}^{\prime}, t^{\prime}\right)}\right|_{f \rightarrow 0}, \\
F_{\alpha \beta}\left(\boldsymbol{r}_{0} \mid \boldsymbol{r}, \boldsymbol{r}^{\prime}, t, t^{\prime}\right) & =\left\langle w_{\alpha}\left(\boldsymbol{r}_{0} \mid \boldsymbol{r}, t\right) w_{\beta}\left(\boldsymbol{r}_{0} \mid \boldsymbol{r}^{\prime}, t^{\prime}\right)\right\rangle .
\end{aligned}
$$

Physically the Green's function is the mean response of the velocity difference to the action of a vanishingly small forcing. In stationary turbulence these quantities depend on $t^{\prime}-t$ only, and we can denote this time difference as $t$. The quantities satisfy the well known and exact Dyson and Wyld coupled equations. The Dyson equation reads

$$
\begin{aligned}
& {\left[\frac{\partial}{\partial t}-\nu \Delta\right] G_{\alpha \beta}\left(\boldsymbol{r}_{0} \mid \boldsymbol{r}, \boldsymbol{r}^{\prime}, t\right)=G_{\alpha \beta}^{0}\left(\boldsymbol{r}_{0} \mid \boldsymbol{r}, \boldsymbol{r}^{\prime}, 0^{+}\right) \delta(t)} \\
& \quad+\int d \boldsymbol{r}_{2} G_{\alpha \delta}^{0}\left(\boldsymbol{r}_{0} \mid \boldsymbol{r}, \boldsymbol{r}_{2}, 0^{+}\right) \int d \boldsymbol{r}_{1} \int_{0}^{t} d t_{1} \\
& \quad \times \Sigma_{\delta \gamma}\left(\boldsymbol{r}_{0} \mid \boldsymbol{r}_{2}, \boldsymbol{r}_{1}, t_{1}\right) G_{\gamma \beta}\left(\boldsymbol{r}_{0} \mid \boldsymbol{r}_{1}, \boldsymbol{r}^{\prime}, t-t_{1}\right),
\end{aligned}
$$

where $G_{\alpha \beta}^{0}\left(\boldsymbol{r}_{0} \mid \boldsymbol{r}, \boldsymbol{r}^{\prime}, 0^{+}\right)$is the bare Green's function determined by Eq. (3.20) in Paper I. The Wyld equation has the form

$$
\begin{aligned}
& F_{\alpha \beta}\left(\boldsymbol{r}_{0} \mid \boldsymbol{r}, \boldsymbol{r}^{\prime}, t\right)=\int d \boldsymbol{r}_{1} d \boldsymbol{r}_{2} \int_{0}^{\infty} d t_{1} d t_{2} G_{\alpha \gamma}\left(\boldsymbol{r}_{0} \mid \boldsymbol{r}, \boldsymbol{r}_{1}, t_{1}\right) \\
\times & {\left[D_{\gamma \delta}\left(\boldsymbol{r}_{1}-\boldsymbol{r}_{2}, t-t_{1}+t_{2}\right)+\Phi_{\gamma \delta}\left(\boldsymbol{r}_{0} \mid \boldsymbol{r}_{1}, \boldsymbol{r}_{2}, t-t_{1}+t_{2}\right)\right] } \\
\times & G_{\delta \beta}\left(\boldsymbol{r}_{0} \mid \boldsymbol{r}^{\prime}, \boldsymbol{r}_{2}, t_{2}\right) .
\end{aligned}
$$

In Eq. (13) the "mass operator" $\Sigma$ is related to the "eddy viscosity" whereas in Eq. (14) the "mass operator" $\Phi$ is the renormalized "nonlinear" noise which arises due to turbulent excitations. Both these quantities are dependent on the Green's function and the correlator, and thus the equations are coupled.

The main result of Paper I is a demonstration of the property of "locality" in the Dyson and Wyld equations. This property means that given a value of $\left|\boldsymbol{r}-\boldsymbol{r}_{0}\right|$ in Eq. (13), the important contribution to the integral on the RHS comes from that region where $\left|\boldsymbol{r}_{1}-\boldsymbol{r}_{0}\right|$ and $\left|\boldsymbol{r}_{2}-\boldsymbol{r}_{0}\right|$ are of the order of $\left|\boldsymbol{r}-\boldsymbol{r}_{0}\right|$. In other words, all the integrals converge both in the upper and the lower limits. The same is true for the Wyld equation, meaning that in the limit of large $L$ and small $\eta$ these length scales disappear from the theory, and there is no natural cutoff in the integrals in the perturbative theory. In this case one cannot form a dimensionless parameter like $L / r$ or $r / \eta$ to carry dimensionless corrections to the K41 scaling exponents. For $\eta \ll\left|\boldsymbol{r}-\boldsymbol{r}_{0}\right| \ll L$ scale invariance prevails, and one finds precisely the K41 scaling exponents:

$$
\begin{aligned}
G_{\alpha \beta}\left(\lambda \boldsymbol{r}_{0} \mid \lambda \boldsymbol{r}, \lambda \boldsymbol{r}^{\prime}, \lambda^{z} t\right) & =\lambda^{\beta_{2}} G_{\alpha \beta}\left(\boldsymbol{r}_{0} \mid \boldsymbol{r}, \boldsymbol{r}^{\prime}, t\right), \\
F_{\alpha \beta}\left(\lambda \boldsymbol{r}_{0} \mid \lambda \boldsymbol{r}, \lambda \boldsymbol{r}^{\prime}, \lambda^{z} t\right) & =\lambda^{\zeta_{2}} F_{\alpha \beta}\left(\boldsymbol{r}_{0} \mid \boldsymbol{r}, \boldsymbol{r}^{\prime}, t\right) .
\end{aligned}
$$

One can derive two scaling relations which hold order by order, i.e.

$$
2 z+\zeta_{2}=2, \quad z+2 \zeta_{2}=2 .
$$

The solution is $z=\zeta_{2}=2 / 3$. It was also shown that the scaling exponent of the Green's function (15) is $\beta_{2}=-3$. Extending such considerations to the higher order structure functions leads to the order-by order K41 prediction that $\zeta_{n}=n / 3$.

Of course, the order by order result (15) which leads to (16) is not necessarily the correct one. If one could resum all the diagrammatic expansion one could find nonperturbative answers that may be different. The whole sum of diagrams may diverge when the outer scale goes to infinity or the inner scale to zero, allowing a renormalization scale to creep in even though the order-by-order theory is convergent. The difficulty is that no one knows how to resum the infinite expansion which exhibits no obvious small parameter.

In this paper we will propose a way out of this difficulty. The new thinking is based on the fusion rules. Instead of considering fully unfused correlation functions only, we will allow some coordinates to be much closer together, say within a distance $\boldsymbol{r}$, whereas the rest will be separated by a much larger distance, say of the order of $\boldsymbol{R}$ where $r \ll R$. We will show that we can form a dimensionless ratio with $R / r$, and that such ratios carry anomalous exponents that are going to survive the process of fusion of coordinates in correlation functions when we make structure functions. We will thus be able to recognize the anomalous exponents even though at first sight there is no obvious renormalization scale.

To clarify how this mechanism works we need to remind ourselves of the appearance of ladder diagrams in the theory of correlations functions. Such diagrams appear in the most transparent way in nonlinear Green's functions, and we review briefly our past results on these objects.

\section{B. The Nonlinear Green's Functions}

The nonlinear Green's function $G_{2,2}\left(\boldsymbol{r}_{0} \mid x_{1}, x_{2}, x_{3}, x_{4}\right)$ describes the response of a product of two velocity fields taken at different space-time coordinates to the action of two forces $f$ :

$$
G_{2,2}^{\alpha \beta \gamma \delta}\left(\boldsymbol{r}_{0} \mid x_{1}, x_{2}, x_{3}, x_{4}\right)=\left\langle\frac{\delta^{2} w_{\alpha}\left(\boldsymbol{r}_{0} \mid x_{1}\right) w_{\gamma}\left(\boldsymbol{r}_{0} \mid x_{2}\right)}{\delta f_{\beta}\left(\boldsymbol{r}_{0} \mid x_{3}\right) \delta f_{\delta}\left(\boldsymbol{r}_{0} \mid x_{4}\right)}\right\rangle,
$$

where for brevity we use the notation $x_{j} \equiv\left\{\boldsymbol{r}_{j}, t_{j}\right\}$. Similarly, one defines the nonlinear Green's functions $\boldsymbol{G}_{p, p}$ as the response of the product of $p$ velocity differences between $p$ distinct points and $\boldsymbol{r}_{0}$ to the action of $p$ forces in different points.

In a Gaussian theory (which ours is not) $\boldsymbol{G}_{2,2}$ would be the products of the linear Green's functions like $G^{\alpha \beta}\left(\boldsymbol{r}_{0} \mid x_{1}, x_{3}\right) G^{\gamma \delta}\left(\boldsymbol{r}_{0} \mid x_{2}, x_{4}\right)$. In a non-Gaussian theory 
it is natural to assume that this quantity is a homogeneous function of its arguments when they are in the scaling regime. This means that

$$
\begin{aligned}
& G_{2,2}^{\alpha \beta \gamma \delta}\left(\boldsymbol{r}_{0} \mid \lambda \boldsymbol{r}_{1}, \lambda^{z} t_{1}, \lambda \boldsymbol{r}_{2}, \lambda^{z} t_{2}, \lambda \boldsymbol{r}_{3}, \lambda^{z} t_{3}, \lambda \boldsymbol{r}_{4}, \lambda^{z} t_{4}\right) \\
= & \lambda^{\beta_{4}} G_{2}^{\alpha \beta \gamma \delta}\left(\boldsymbol{r}_{0} \mid x_{1}, x_{2}, x_{3}, x_{4}\right)
\end{aligned}
$$

From the Gaussian decomposition of this quantity we would guess that $\beta_{4}=2 \beta_{2}=-6$. The proof of locality in Paper I means that there is no perturbative mechanism to change this scaling index. On the other hand, this quantity, which is a function of four space-time coordinates $x_{i}$ has scaling properties that are not exhausted by the overall scaling exponent $\beta_{4}$. We have shown in Paper II that when we consider its dependence on ratios of spacetime coordinates in their asymptotic regimes w e pick up a set of anomalous scaling exponents. The main result of Paper II was that in the regime $r_{1} \sim r_{2} \ll r_{3} \sim r_{4}$ the diagrammatic expansion of this object produces logarithms like $\ln \left(r_{3} / r_{1}\right)$ to some power. It was explained that the sum of such logarithmically large contributions is given by $\left(r_{3} / r_{1}\right)^{\Delta}$ with some anomalous exponent $\Delta$. To make the appearance of anomalous exponents evident we review the simplest object that resums to logarithms, i.e. the series of "ladder diagrams".

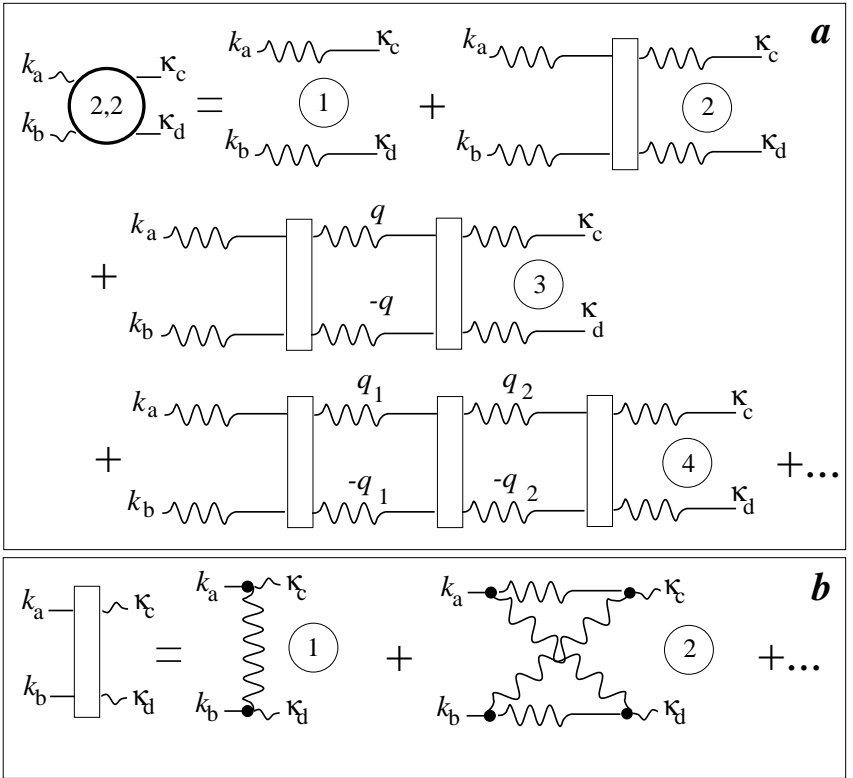

FIG. 2. Diagrams for $\mathbf{G}_{2,2}\left(\mathbf{k}_{a}, \omega_{a}, \mathbf{k}_{b}, \omega_{b}, \kappa_{c}, \omega_{c}, \kappa_{d}, \omega_{d}\right)$ (Panel (a)) and of the rung $\mathbf{R}\left(\mathbf{k}_{a}, \mathbf{k}_{b}, \kappa_{c}, \kappa_{d}\right)$ (Panel (b)). Diagram (1) in Panel (a) is the Gaussian contribution made of a product of two linear Green's functions. Diagram (2) is the skeleton contribution, and diagrams (3) and (4) are the 1-loop and 2-loop contributions respectively. In Panel (b) we show the beginning of the infinite series expansion for the rung, with diagram (1) being the bare rung.

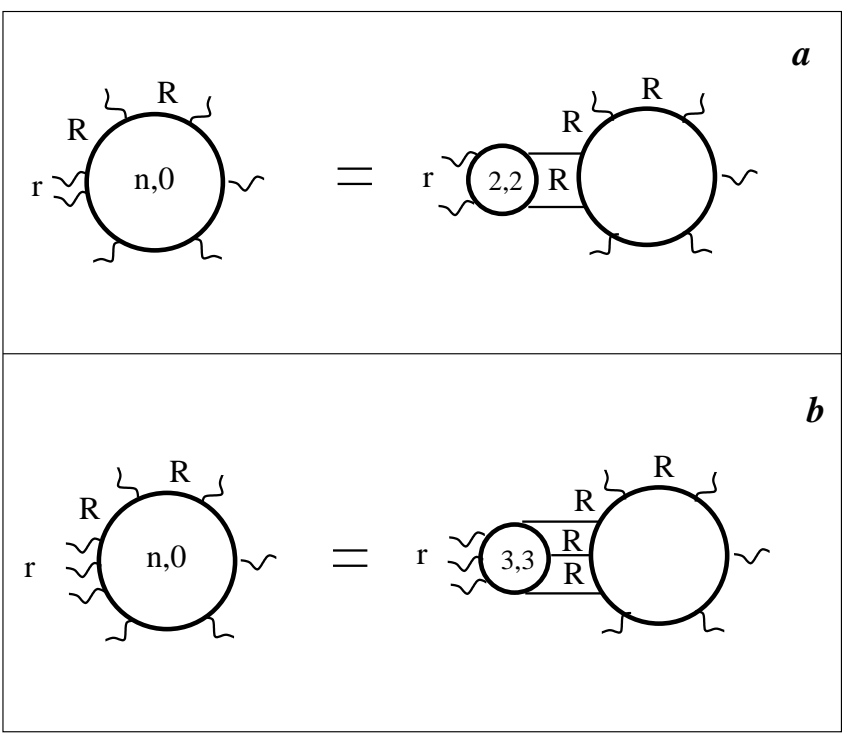

FIG. 3. A diagrammatic representation of the fusion process. A typical $n$ the order correlation function is represented by $n$ wavy lines decorating a circle. Panel (a): The fusion of 2 coordinates to within a distance $r$ which is much smaller that the typical separations $R$ between the other coordinates. Panel (b): The fusion of three coordinates to within a distance $r \ll R$ from each other. The availability of all the possible diagrams afforded by the topological rules allows us to expose a contribution that is precisely $G_{p, p}$ with $p=2$ and 3 respectively.

The diagrammatic representation of the nonlinear Green's function (17) is shown in Fig. 2a, where the notation of the diagrams is explained shortly in the figure legend and at length in Appendix A. The 4-point "rungs" appearing in the ladders were represented in Paper II as an infinite series expansion whose beginning is shown in Fig. 2 $\mathrm{b}$. The rest of the expansion involves exceedingly complicated diagrams that we failed to resum analytically. Nevertheless, just from general properties one could show that the ladder with $n$ rungs contains a contribution of order $\left[\Delta \ln \left(r_{3} / r_{1}\right)\right]^{n} / n$ !. The summation of all these contributions gives a term proportional to $\left(r_{3} / r_{1}\right)^{\Delta}$, and this is the observation that we want to build on in this paper.

\section{LADDER DIAGRAMS IN $N$ 'TH ORDER CORRELATION FUNCTIONS}

In this Section we demonstrate how the anomalous exponents of higher order correlation functions can be related to resummed ladder diagrams. The idea is to consider a typical $n$th order correlation function and to almost fuse $p$ coordinates, $p<n$, chosen from the available $n$ coordinates. The point to observe is that the diagrammatic theory allows us to write, upon observation, all the topologically possible diagrams appearing in the expansion of a given object. Thus for example consider Fig. 3 
where we represent a general $n$th order correlation function, in which two coordinates are a distance $r$ from each other, and all the rest are a distance $R$ from them and from each other. While coalescing the two coordinates we pull out a contribution that reads $G_{2,2}$ connected to the rest of the diagram with $n-2$ coordinates. We know that this contribution exists since it is allowed topologically. But this fragment is represented in its turn by the sum of the ladder diagrams that we present in Fig. 2. We thus state that whenever we are about to fuse two coordinates in any $n$th order correlation function we can expose a series of diagrams that are the same as those that appear in the expansion of the nonlinear Green's function $G_{2,2}\left(\boldsymbol{r}_{0} \mid x_{1}, x_{2}, x_{3}, x_{4}\right)$, together with the logarithmic divergences that are associated with them. In Appendix A we explain that in doing so we really take into account all the necessary contributions, leaving nothing uncontrolled.

Now we employ the fusion rules. These tell us that as a function of the two fusing coordinates the $n$th order correlation function is a homogeneous function whose homogeneity exponent is $\zeta_{2}$. Accordingly, if we succeed to resum the ladder diagrams in the limit $r \ll R$ we should find an anomalous part of $\zeta_{2}$ from the dimensionless power $(R / r)^{\Delta}$.

Similarly, we can almost fuse 3,4 or $p$ coordinates, and accordingly pull out of the diagram for the $n$th order correlation functions more complex ladders with 3, 4 or $p$ struts. For example in Fig. $3 \mathrm{~b}$ we show how the fusion of 3 coordinates singles out a fragment that is $G_{3,3}$ whose ladder diagrams have three struts cf. Fig. 4. The fusion rules guarantee that the $n$th order correlation is a homogeneous function of the $p$ fusing coordinates with $\zeta_{p}$ being the homogeneity exponent. We will show that these more complex ladders also resum into power laws in $R / r$, being responsible for the anomalous parts of $\zeta_{p}$.

At this point all this is a bit formal, since we do not have an explicit form of the rungs in the ladder diagrams, and we can compute nothing without this knowledge. In the next two Sections we will address this issue and demonstrate that a judicious use of the fusion rules dictates enough knowledge of the rungs to take us through a useful calculation.

\section{BUILDING THE THEORY ON THE BACKGROUND OF K41}

In this Section we reorganize the theory such that Kolmogorov's 41 theory serves as its "free" limit. In other words, we aim at achieving a theory in which resummations of divergent contributions would directly give the anomalous parts of the scaling exponents; the K41 parts should be obvious order-by-order. This is done in two steps, that are correspondingly presented in Subsects. IV A and IVB.

\section{A. Resummation into K41 propagators}

It was explained in Subsect. II A that our theory is developed in the BL-representation, to eliminate spurious IR divergences that stem from the sweeping interactions. The main result of Paper I was that after lineresummation each diagram in the BL-diagrammatic expansion of the propagators (Green's function and double correlation function) converged in the infrared and the ultraviolet regimes. Accordingly, K41 scaling is a solution of the order-by-order theory. Nevertheless, the propagators in the BL-representation lose translational invariance, and are therefore not diagonal in Fourier space. For the purpose of actual calculations it is extremely advantageous to rearrange the theory such that the BLpropagators become again diagonal in Fourier space.

The actual resummation that is necessary is presented in Appendix B. It results in a diagrammatic theory that is topologically exactly the same as the standard Wyld diagrammatic expansion before line resummation. There are two differences as explained in the Appendix B. For the purposes of our considerations below the main issue is the simple form of the the propagators that appear as lines in the diagrams: they exhibit K41 scaling exponents:

$$
\begin{aligned}
& G_{\alpha \beta}(\boldsymbol{k}, \omega)=P_{\alpha \beta}(\boldsymbol{k}) g(k, \omega), g(k, \omega)=\frac{1}{\omega+i \gamma(k)} \\
& F_{\alpha \beta}(\boldsymbol{k}, \omega)=P_{\alpha \beta}(\boldsymbol{k}) f(k, \omega), f(k, \omega)=\frac{\phi(k)}{\omega^{2}+\gamma^{2}(k)} .
\end{aligned}
$$

In these formulae the scaling exponents are carried by

$$
\gamma(k)=c_{\gamma} \bar{\epsilon}^{1 / 3} k^{2 / 3}, \quad \phi(k)=c_{\phi} \bar{\epsilon} k^{-3},
$$

where $c_{\gamma}$ and $c_{\phi}$ are dimensionless constants.

\section{B. Renormalization to K41 4-point rung}

In this Subsection we determine the form of the 4point rungs of the ladder diagrams in two steps. These two steps are based on the following observation: the diagrammatic expansion of the rung includes many diagrams, some of which contain in them additional subsets of ladder diagrams. In the first step we will consider the rungs as if all the diagrams appearing in their infinite series were resummed, except for their own internal subsets of ladder diagrams. In the second step we will consider also the ladder diagrams appearing in the series for the rung. We aim to a situation in which all the ladders that appear in the theory, like in Fig. 2, contain already renormalized rungs. However, instead of evaluating the rungs from actual resummations we are going to determine their form using the fusion rules. Thus in the first 
step we find the form of the rung that results, upon fusion, in K41 scaling exponents. In the second step we recognize that the rungs themselves have ladders, leading to an anomalous correction in the scaling properties of the rungs themselves. This being accomplished, we will have our final form of the rung. Then we turn to the ladder diagrams appearing in the fused correlation functions, using the rung as a basic building block of the theory. All anomalies of all the measurable statistical objects will result from resummations of the remaining ladder diagrams.

Consider Fig. 2a a, in which the rung appears as an object. It is given in terms of an infinite series of diagrams in Fig. 2 2 b. It is in fact a 4-point vertex depending on four $\boldsymbol{k}$-vectors and four frequencies. As a first step we consider the value of the rung when all the frequencies are zero, denoting it in this limit as $\boldsymbol{R}\left(\boldsymbol{k}_{a}, \boldsymbol{k}_{b}, \boldsymbol{\kappa}_{c}, \boldsymbol{\kappa}_{d}\right)$. At a later point we will explain that this is sufficient for our purposes. The bare value of this object can be read directly from diagram (1) in Fig. 2bo, with two bare BL-vertices $\boldsymbol{\Gamma}$ and one double correlation function. The answer is

$$
\begin{aligned}
& R_{0}^{\alpha \beta \gamma \delta}\left(\boldsymbol{k}_{a}, \boldsymbol{k}_{b}, \boldsymbol{\kappa}_{c}, \boldsymbol{\kappa}_{d}\right) \\
& =\delta_{0} \bar{\epsilon}^{1 / 3} \frac{\Gamma^{\alpha \gamma \sigma}\left(\boldsymbol{k}_{a}, \boldsymbol{\kappa}_{c}, \boldsymbol{k}_{e}\right) \Gamma^{\beta \delta \sigma}\left(\boldsymbol{k}_{b}, \boldsymbol{\kappa}_{d},-\boldsymbol{k}_{e}\right)}{k_{e}^{13 / 3}},
\end{aligned}
$$

where $\boldsymbol{k}_{e} \equiv \boldsymbol{k}_{a}-\boldsymbol{\kappa}_{c}=\boldsymbol{\kappa}_{d}-\boldsymbol{k}_{b}$, and $\delta_{0}$ is a dimensionless constant.

We demonstrate now that if we use this bare form of the rung the fusion rules would predict dissipative exponents, $\zeta_{n}=n$. We first demonstrate this in the context of $\zeta_{2}$. Consider a general $n$ order correlation function as in Fig. Ba, and fuse two coordinates, pulling out the fragment of $\boldsymbol{G}_{2,2}$ as shown on the RHS of Fig. 3a. We will now compute the scaling exponent by finding the $r$ dependence of this fragment when the two coordinates approach each other to a small distance $r \ll R$ where $R$ is the typical distance between all the other coordinates. To find the $r$ dependence we must integrate according to the explanation in Appendix $\mathbf{Q}$, and to this aim we introduce the object $T_{2}(r, \boldsymbol{\kappa})$ :

$$
\begin{aligned}
& T_{2}(r, \boldsymbol{\kappa})=\int \frac{d \boldsymbol{k}_{a}}{(2 \pi)^{3}} 4 \sin ^{2}\left(\frac{1}{2} \boldsymbol{k}_{a} \cdot \boldsymbol{r}\right) \\
& \times \int \frac{d \omega_{a}}{2 \pi} G_{2,2}\left(\boldsymbol{k}_{a}, \omega_{a},-\boldsymbol{k}_{a},-\omega_{a}, \boldsymbol{\kappa}, 0,-\boldsymbol{\kappa}, 0\right) .
\end{aligned}
$$

We are interested in the $r$ dependence of this object in the limit $r \kappa \ll 1$. To calculate $T_{2}(r, \boldsymbol{\kappa})$ in this limit we return to Fig. 2a. Obviously the Gaussian contribution diagram (1) is irrelevant in this limit. The skeleton diagram (2) contributes the following integral:

$$
\begin{aligned}
& T_{2}^{\mathrm{s}}(r, \boldsymbol{\kappa}) \approx \int \frac{d \omega_{a}}{2 \pi} \int \frac{d \boldsymbol{k}_{a}}{(2 \pi)^{3}} 4 \sin ^{2}\left(\frac{1}{2} \boldsymbol{k}_{a} \cdot \boldsymbol{r}\right) \\
& \times g\left(k_{a}, \omega_{a}\right) g\left(-k_{a},-\omega_{a}\right) R_{0}\left(\boldsymbol{k}_{a},-\boldsymbol{k}_{a}, \boldsymbol{\kappa},-\boldsymbol{\kappa}\right),
\end{aligned}
$$

where the tensor indices of the rung were contracted for the longitudinal contribution. The superscript "s" is used here and below to denote skeleton contributions. We note that in the limit $k \gg \kappa$ the BL vertices are proportional to the smallest wavevector $\kappa$. Thus the rung is proportional to $\kappa^{2} / k_{a}^{13 / 3}$. Integrating over the frequencies of the two Green's functions $g\left(k_{a}, \omega_{a}\right)$ in this rung [cf. Eqs. 119, 21)] results in the evaluation $1 /\left[\gamma\left(k_{a}\right) k_{a}^{13 / 3}\right] \propto k_{a}^{-5}$. Thus the $r$ dependence of $T_{2}^{\mathrm{s}}$ is given by

$$
T_{2}^{\mathrm{s}}(r, \boldsymbol{\kappa}) \propto \int \frac{d \boldsymbol{k}_{a}}{(2 \pi)^{3}} 4 \sin ^{2}\left(\frac{1}{2} \boldsymbol{k}_{a} \cdot \boldsymbol{r}\right) \frac{1}{k_{a}^{5}} .
$$

Up to logarithmic corrections this integral is proportional to $r^{2}$ which is the dissipative solution. Similarly, if we use the bare rung in the diagram in Fig. 2 b to determine $\zeta_{3}$ we will find $\zeta_{3}=3$. In general we will find $\zeta_{n}=n$ instead of the K41 value of $n / 3$. Now one could think that the correct values of the inertial range exponents may be obtained from resumming all the ladder diagrams with the bare rungs. This was the point of view proposed in Paper I. In such a case the sought after correction to the scaling exponents is of the order of unity, and it is unclear how to develop a controlled resummation. In this paper we point out a new way, based on the existence of a renormalized rung which gives, upon fusion, K41 exponents before ladder resummations. The characteristics of the renormalized rung in such a scheme are dictated by the fusion rules.

Next we want to determine the renormalized form of the rung. To this aim we repeat the exercise of integrating over the two Green's functions and the rung, with the vertices determined as before in the limit $k_{a} \approx k_{b} \gg$ $\kappa_{c} \approx \kappa_{d}$. But now we leave the exponent of $k_{a}$ in the asymptotic evaluation of the rung free, and demand that the result of the integration will be $k_{a}^{-5 / 3}$. We find that this requires that $R_{0} \propto k_{a}^{-3}$.

We are now in a position to propose a renormalized form of the rung which in a proper calculation could be obtained by summing up all the non-ladder diagrams that contribute to this rung. This conforms with our basic hypothesis that all non-ladder diagrams contribute to K41, whereas the ladders are responsible for the anomalous scaling. Since K41 does not allow $L$ renormalization we propose the form

$$
\begin{aligned}
& R^{\alpha \beta \gamma \delta}\left(\boldsymbol{k}_{a}, \boldsymbol{k}_{b}, \boldsymbol{\kappa}_{c}, \boldsymbol{\kappa}_{d}\right) \\
& =\delta \bar{\epsilon}^{1 / 3} \frac{\Gamma^{\alpha \gamma \sigma}\left(\boldsymbol{k}_{a}, \boldsymbol{\kappa}_{c}, \boldsymbol{k}_{e}\right) \Gamma^{\beta \delta \sigma}\left(\boldsymbol{k}_{b}, \boldsymbol{\kappa}_{d},-\boldsymbol{k}_{e}\right)}{k_{e}^{3} \kappa_{c}^{2 / 3} \kappa_{d}^{2 / 3}} .
\end{aligned}
$$

This form gives the K41 overall scaling exponent (which is the same as in the bare rung $\boldsymbol{R}_{0}$, and in addition agrees with the fusion rules for the second order correlation function with a K41 scaling exponent. In addition it is symmetric, as it should be, for exchanging the indices $a$ and $b$ together with $c$ and $d$. Note that $\delta$ is now 
a renormalized unknown dimensionless parameter which will be determined later.

To proceed, we note that our actual calculation (see below) depends really only on the asymptotic properties of the rung, which are rigidly determined by the fusion rules. We can thus attempt to simplify the form of the rung as much as possible, preserving the asymptotic and parity properties unchanged. In particular we note that the BL vertices $\boldsymbol{\Gamma}\left(\boldsymbol{k}_{a}, \boldsymbol{\kappa}_{c}, \boldsymbol{k}_{e}\right)$ have complicated structure which makes calculations involving them rather difficult. Therefore we propose to use instead Eulerian vertices $\boldsymbol{V}\left(\boldsymbol{k}_{a}, \boldsymbol{\kappa}_{c}, \boldsymbol{k}_{e}\right)$ corrected by a factor $-2\left(\boldsymbol{k}_{b} \cdot \boldsymbol{\kappa}_{c}\right) /\left[k_{a}^{2}+k_{b}^{2}+\kappa_{c}^{2}\right]$. The correction is aimed at reproducing the asymptotic behavior of the BL vertex $\Gamma^{\alpha \gamma \sigma}\left(\boldsymbol{k}_{a}, \boldsymbol{\kappa}_{c}, \boldsymbol{k}_{e}\right) \sim \min \left\{k_{a}, k_{b}, \kappa_{c}\right\}$. Thus instead of 26) one has

$$
\begin{gathered}
R^{\alpha \beta \gamma \delta}\left(\boldsymbol{k}_{a}, \boldsymbol{k}_{b}, \boldsymbol{\kappa}_{c}, \boldsymbol{\kappa}_{d}\right)=\frac{-4 \delta \bar{\epsilon}^{1 / 3}\left(\boldsymbol{\kappa}_{c} \cdot \boldsymbol{k}_{e}\right)\left(\boldsymbol{\kappa}_{d} \cdot \boldsymbol{k}_{e}\right)}{\left[k_{a}^{2}+\kappa_{c}^{2}+k_{e}^{2}\right]\left[k_{b}^{2}+\kappa_{d}^{2}+k_{e}^{2}\right]} \\
\times \frac{V^{\alpha \gamma \sigma}\left(\boldsymbol{k}_{a}, \boldsymbol{\kappa}_{c}, \boldsymbol{k}_{e}\right) V^{\beta \delta \sigma}\left(\boldsymbol{k}_{b}, \boldsymbol{\kappa}_{d},-\boldsymbol{k}_{e}\right)}{k_{e}^{3}\left(\kappa_{c} \kappa_{d}\right)^{2 / 3}} .
\end{gathered}
$$

As a further simplification of the actual calculations we will use a 1-dimensional reduction of the problem (preserving the asymptotic scaling properties and parity) in which instead of 3-dimensional integrations $\int d^{3} k /(2 \pi)^{3}$ we will use 1 -dimensional one $\int_{-\infty}^{\infty} d k / 2 \pi$. Then we can disregard the vector indices and replace $V^{\alpha \gamma \sigma}\left(\boldsymbol{k}_{a}, \boldsymbol{\kappa}_{c}, \boldsymbol{k}_{e}\right) \rightarrow k_{a},\left(\boldsymbol{k} \cdot \boldsymbol{k}^{\prime}\right) \rightarrow k k^{\prime}$ (keeping the signs) and $k_{e}^{3}$ in the denominator by $\left|k_{e}\right|$ (because we replaced 3 -d by 1-d integration). The 1-dimensional version of the rung (27) turns into

$R\left(k_{a}, k_{b}, \kappa_{c}, \kappa_{d}\right)=\frac{-4 \delta \bar{\epsilon}^{1 / 3} k_{a} k_{b} \kappa_{c} \kappa_{d}\left|k_{e}\right|}{\left[k_{a}^{2}+\kappa_{c}^{2}+k_{e}^{2}\right]\left[k_{b}^{2}+\kappa_{d}^{2}+k_{e}^{2}\right]\left|\kappa_{c} \kappa_{d}\right|^{2 / 3}}$.

Note that here $k_{a}, k_{b}, \kappa_{c}, \kappa_{d}$ are in the interval $\pm \infty$ and that they carry signs in order to preserve the parity of the rungs. $k_{a}, k_{b}$ are incoming wave vectors and $\kappa_{c}, \kappa_{d}$ are outgoing, and they conserve momentum,

$$
k_{a}+k_{b}=\kappa_{c}+\kappa_{d} .
$$

Substituting into (28) $k_{e}=k_{a}-\kappa_{c}=k_{b}-\kappa_{d}$ one gets finally:

$$
\begin{aligned}
& R\left(k_{a}, k_{b}, \kappa_{c}, \kappa_{d}\right) \\
= & -\delta \bar{\epsilon}^{1 / 3} \frac{k_{a} k_{b} \kappa_{c} \kappa_{d}\left|k_{a}-\kappa_{c}\right|}{\left[k_{a}^{2}-k_{a} \kappa_{c}+\kappa_{c}^{2}\right]\left[k_{b}^{2}-k_{b} \kappa_{d}+\kappa_{d}^{2}\right]\left|\kappa_{c} \kappa_{d}\right|^{2 / 3}} .
\end{aligned}
$$

In particular,

$$
\begin{aligned}
R(k,-k, \kappa,-\kappa) & =\frac{-\delta \bar{\epsilon}^{1 / 3} k^{2} \kappa^{2 / 3}|k-\kappa|}{\left[k^{2}-k \kappa+\kappa^{2}\right]^{2}}, \\
R\left(k,-k, \kappa, \kappa^{\prime}\right) & =\frac{\delta \bar{\epsilon}^{1 / 3} \operatorname{sign}\left(\kappa \kappa^{\prime}\right)\left|\kappa \kappa^{\prime}\right|^{1 / 3}}{|k|} \\
\text { for } \kappa, \kappa^{\prime} & \ll k .
\end{aligned}
$$

To check that we get the right K41 scaling exponents with the new renormalized rung we need to recalculate the 1-dimensional version of Eq. (24) with (30) for the rung:

$$
\begin{aligned}
T_{2}^{\mathrm{s}}(r, \kappa) & =\int_{-\infty}^{\infty} \frac{d k}{2 \pi} 4 \sin ^{2}\left(\frac{k r}{2}\right) R(k,-k, \kappa,-\kappa) \\
& \times \int_{-\infty}^{\infty} \frac{d \omega}{2 \pi} g(k, \omega) g(-k,-\omega) .
\end{aligned}
$$

Using Eq. (19) the frequency integral yields $-1 / 2 \gamma(k)$. Thus

$$
T_{2}^{\mathrm{S}}(r, \kappa)=\frac{\delta \kappa^{2 / 3}}{c_{\gamma} \pi} \int_{-\infty}^{\infty} d k \frac{|k|^{4 / 3}|k-\kappa| \sin ^{2}\left(\frac{k r}{2}\right)}{\left[k^{2}-k \kappa+\kappa^{2}\right]^{2}} .
$$

In the limit $\kappa \rightarrow 0$ the integral simplifies to

$$
T_{2}^{\mathrm{s}}(r, \kappa)=2 \tilde{\delta} \kappa^{2 / 3} \int_{0}^{\infty} \frac{d k}{k^{5 / 3}} \sin ^{2}\left(\frac{k r}{2}\right)
$$

where

$$
\tilde{\delta} \equiv \frac{\delta}{\pi c_{\gamma}}
$$

This integral is elementary, reading

$$
T_{2}^{\mathrm{s}}(r, \kappa)=-\frac{1}{2} \tilde{\delta}(\kappa r)^{2 / 3} \Gamma\left(-\frac{2}{3}\right) \approx 2 \tilde{\delta}(\kappa r)^{2 / 3}
$$

where $\Gamma(x)$ is the gamma-function.

The point to notice is that in the asymptotic limit $\kappa \rightarrow 0$ the only properties of the rung that guaranteed the appearance of the scaling exponent $2 / 3$ are the asymptotic properties that we preserved in the series of simplifications leading to (30). In general, we will show below that the series of simplifications of the model form of the rung are of absolutely no import also for the calculation of the anomalous scaling exponents up to 1-loop order. We will show below that we get precisely the same exponents in this order with any arbitrary analytic form of the rung, with tensor indices or without, in 3-d form or 1-d form or whatever, as long as the asymptotics are preserved, as they are. In 2-loop order this is no longer true. The actual numbers obtained in the 2-loop order are model dependent. We will show however that the sensitivity of the predicted exponents $\zeta_{n}$ to the model for the rung is small as long as $n<8$. We need the 2loop order mainly to make sure that it corrects for some unacceptable properties of the 1-loop results for higher order correlation functions. 

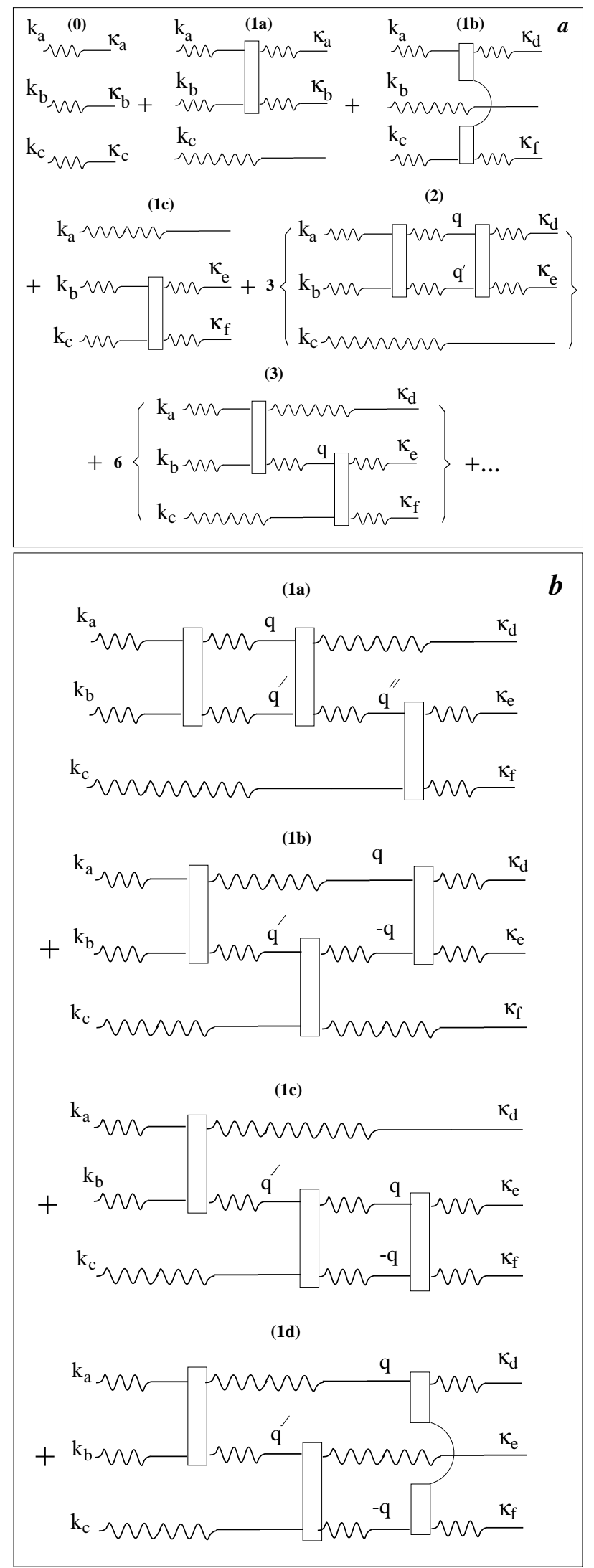

FIG. 4. Diagrammatic expansion of $\mathbf{G}_{3,3}$ (panel a): Contributions with no rungs, (diagram 0), one rung and two rungs. (panel b): Contributions with three rungs.
Before we proceed we need to check the self consistency of our approach. We need to make sure that all higher order non-linear Green's function $\boldsymbol{G}_{p, p}$ (the response of $p$ velocities to $p$ forcing) yield, upon fusion, the correct K41 exponent for $p$ th order correlation functions $\zeta_{p}^{\mathrm{K} 41}=p / 3$. For this aim we have to consider the so called skeleton diagrams which are the lowest order connected diagrams without loops. For $\boldsymbol{G}_{2,2}$ this is diagram (2) in Fig. 2a. for $\boldsymbol{G}_{3,3}$ the skeleton contribution are shown as diagrams (3) on Fig. Ga. We must make sure that the skeleton diagrams, upon fusion, yield K41 scaling $\zeta_{p}^{\mathrm{K} 41}=p / 3$ for the appropriate $p^{\text {th }}$-order correlation functions, since our grand hypothesis is that the anomalous scaling comes only from ladder resummations.

This test of self consistency is presented in Appendix Q. The important conclusion of this Appendix is that the skeleton diagrams for $G_{p, p}\left(\left\{k_{j}, \kappa_{j}\right\}\right.$ ) (with asymptotics of the rung defined by the two-point fusion rules with $\zeta_{2}^{\mathrm{K} 41}=2 / 3$ ) automatically reproduces the K41 scaling exponent $\zeta_{p}^{\mathrm{K} 41}=p / 3$ when $p$ points are fused.

\section{SANDING THE FLOOR IN THE ONE-LOOP ORDER}

In this Section we demonstrate the most important new property of the resummed theory, i.e. that the rungs in the ladder diagrams appear with a small parameter. This will allow us to develop a controlled ladder resummation, something that to our knowledge has never been available before. In fact, we will lay out in this Section all that is needed to calculate the scaling exponents in the 1-loop order. In Subsect. $\mathrm{VA}$ we demonstrate that the prefactor $\delta$ of the rung is the order of $\delta_{2}$ which is the anomalous part of $\zeta_{2}$ and thus small. In Subsect. VB we consider the anomalous exponent of the rung itself, denoted as $\delta_{a}$, and stemming from ladder resummations within the rung infinite series representation. In Subsect. $\mathrm{VC}$ we reconsider the contribution of the skeleton diagrams to the scaling exponents upon fusion, taking into account the anomaly of the rung. In Subsect. VD we throw in the inputs: the fact that $\zeta_{3}=1$ and the experimental value of $\zeta_{2}$. The result is Eq. (60) which states that in the 1-loop order all the unknown parameters are numerically identical. From this point the calculation of all the other scaling exponents in the 1-loop order is straightforward.

\section{A. The 4-point rung is small!}

Here we show that the coefficient $\delta$ in front of the renormalized 4-point rung (26) is of the order of the correction to $\mathrm{K} 41$ of the scaling exponent $\zeta_{2}$ :

$$
\delta_{2}=\zeta_{2}-\zeta_{2}^{\mathrm{K} 41} \approx 0.03 .
$$


To this aim consider the 1-dimensional version of the quantity $T_{2}(r, \kappa)$ of $(23)$ :

$$
\begin{aligned}
& T_{2}(r, \kappa)=\int_{-\infty}^{\infty} \frac{d k_{a}}{2 \pi} 4 \sin ^{2}\left(\frac{1}{2} k_{a} r\right) \\
& \times \int_{-\infty}^{\infty} \frac{d \omega_{a}}{2 \pi} G_{2,2}\left(k_{a}, \omega_{a},-k_{a},-\omega_{a}, \kappa, 0,-\kappa, 0\right) .
\end{aligned}
$$

We will examine the ratio of the contributions of the 1-loop diagram (3), denoted below as $T_{2}^{(1)}(r, \kappa)$, to the contribution of the skeleton diagram Eq. (33) [diagram (2) in Fig. 22a]. After performing the frequency integrals the 1-dimensional form of $T_{2}^{(1)}(r, \kappa)$ [cf. diagram (3) in Fig. [2a] is:

$$
\begin{aligned}
& T_{2}^{(1)}(r, \kappa)=\int_{-\infty}^{\infty} \frac{d k_{a}}{\pi \gamma\left(k_{a}\right)} \sin ^{2}\left(\frac{1}{2} k_{a} r\right) \\
& \times \int_{-\infty}^{\infty} \frac{d q}{2 \pi \gamma\left(k_{a}\right)} R\left(k_{a},-k_{a}, q,-q\right) R(q,-q, \kappa,-\kappa) .
\end{aligned}
$$

In the asymptotic limit defined by $\kappa r \rightarrow 0$ the main contribution to the integral comes from the 2 symmetric regions of the q-integration in which $|\kappa| \ll|q| \ll\left|k_{a}\right|$. In these regions we use the form (32). We calculate

$$
T_{2}^{(1)}(r, \kappa) \approx 2 \tilde{\delta}^{2} \kappa^{2 / 3} \int_{0}^{\infty} \frac{d k_{a}}{k_{a}^{5 / 3}} \sin ^{2}\left(\frac{1}{2} k_{a} r\right) \int_{\kappa}^{k_{a}} \frac{d q}{q}
$$

As expected the loop integral over $q$ produces a logarithmic contribution. At this point we use the asymptotical identity

$$
\lim _{\kappa r \rightarrow 0} \int_{0}^{\infty} d(k r) f(k r) \ln \left(\frac{k}{\kappa}\right)=\ln \left(\frac{1}{r \kappa}\right) \int_{0}^{\infty} d(k r) f(k r),
$$

which produces, upon comparison with Eq. (35) the final result

$$
T_{2}^{(1)}(r, \kappa)=\tilde{\delta} \ln \left[\frac{1}{\kappa r}\right] T_{2}^{(\mathrm{s})}(r, \kappa)
$$

The factor $\tilde{\delta}$ that was introduced in Eq. (36) reappears here in front of the logarithm as the effective parameter of expansion.

Analogously one computes the leading contribution of the two-loop diagram (4) in Fig. 2a. This is done explicitly in Subsect. VII A and Appendix D:

$$
T_{2}^{(2)}(r, \kappa)=\frac{1}{2}\left[\tilde{\delta} \ln \left(\frac{1}{r \kappa}\right)\right]^{2} T_{2}^{(\mathrm{s})}(r, \kappa)
$$

and, in general the leading contribution of the $n$-loop diagram:

$$
T_{2}^{(n)}(r, \kappa)=\frac{1}{n !}\left[\tilde{\delta} \ln \left(\frac{1}{r \kappa}\right)\right]^{n} T_{2}^{(\mathrm{s})}(r, \kappa) .
$$

The sum of all these contributions is as follows:

$$
\begin{aligned}
T_{2}(r, \kappa) & =T_{2}^{(\mathrm{s})}(r, \kappa)+\sum_{m=1}^{\infty} T_{2}^{(m)}(r, \kappa) \\
& =T_{2}^{(\mathrm{s})}(r, \kappa) \sum_{m=0}^{\infty} \frac{1}{m !}\left[\tilde{\delta} \ln \left(\frac{1}{r \kappa}\right)\right]^{m}=\frac{T_{2}^{(\mathrm{s})}(r, \kappa)}{(r \kappa)^{\tilde{\delta}}} .
\end{aligned}
$$

We see that, as usual, resummation of the leading contributions from the logarithmic ladder diagrams results in the power function with the exponent $\tilde{\delta}$ which is the prefactor of the logarithm in the one-loop diagram, see Eq. (43). Because the expected correction $\delta_{2}$ to the K41 exponent $\zeta_{2}^{\mathrm{K} 41}$ is small ( $\delta_{2} \approx 0.03$ ) we conclude that the prefactor of the rung $\tilde{\delta}$ is small as well! This allows us to begin with the one-loop approximation in computing the higher order scaling exponents $\zeta_{p}$ with $p>2$.

\section{B. Anomalous correction of the rung asymptotics}

So far we have disregarded the explicit appearance of ladder diagrams in the infinite series that defines the rung itself. As pointed in Paper II the same kind of ladder resummation that is responsible for the anomaly of the exponents of the nonlinear Green's functions will also contribute an anomalous part to the scaling properties of the rung. Nevertheless the outer and inner scale do not appear in the rung either, and therefore the anomaly is explicit only in the asymptotic regime where we have a ratio of large and small scales. In this Subsection we flush out this anomaly.

Instead of (32) we expect

$$
\begin{array}{r}
R_{\mathrm{a}}\left(k,-k, \kappa, \kappa^{\prime}\right)=\frac{\delta \bar{\epsilon}^{1 / 3} \operatorname{sign}\left(\kappa \kappa^{\prime}\right)\left|\kappa \kappa^{\prime}\right|^{1 / 3+\delta_{\mathrm{a}}}}{|k|^{1+2 \delta_{\mathrm{a}}}} \\
\text { for } k \gg \kappa, \kappa^{\prime},
\end{array}
$$

with some anomalous exponent $\delta_{\mathrm{a}}$ which is expected (and later demonstrated) to be of the order of $\tilde{\delta}$ as it stems from the same origin. This correction to the asymptotics may be achieved, for example by the following model form of the rung 30 ):

$$
R_{\mathrm{a}}\left(k_{a}, k_{b}, \kappa_{c}, \kappa_{d}\right)=R\left(k_{a}, k_{b}, \kappa_{c} \kappa_{d}\right)\left(\frac{\left|\kappa_{c} \kappa_{d}\right|}{\left|k_{a}-\kappa_{c}\right|^{2}}\right)^{\delta_{\mathrm{a}}} .
$$

As before, we will argue that the exact analytic form of the rung is not important for our calculations, and only the asymptotic scaling form is essential. This statement will be shown to be exact in the 1-loop order. We thus need at this point only to preserve the essential properties, i.e. that the outer scale $L$ cannot appear due to locality, that the rung has to be symmetric with respect $a, b \rightarrow c, d$, etc. 


\section{Contributions of the skeleton diagrams with the anomalous 4-point rung}

In this Subsection we reconsider the skeleton diagrams appearing in the nonlinear Green's functions $G_{p, p}$ but taking into account the anomaly of the rung. In other words we are going to compute the scaling exponents accounting only for the ladder resummation inside the rung, but not the ladder resummation with the anomalous renormalized rungs. This final step will be done in Sects. VI and VII.

We are interested in the scaling exponents of structure functions in $r$ representation, and these are obtained from the correlation functions in $k$ representation as detailed in Appendix Q. Upon fusion we obtain automatically contributions behaving like nonliner Green's functions. Accordingly the objects of interest in the analysis below are the nonlinear Green's functions in which the $k$ dependence of the fusing coordinates is transformed to $r$ representation. The outgoing wavevectors $\kappa$ are left as are, and the outgoing frequencis can be put to zero with impunity. This results in objects defined in a mixed $r, \kappa$ representation, which we denote as $T_{p}\left(r,\left\{\kappa_{j}^{\prime}\right\}\right)$ :

$$
\begin{aligned}
T_{p}\left(r,\left\{\kappa_{j}^{\prime}\right\}\right) & =\int_{-\infty}^{\infty} \prod_{i=1}^{p} \frac{d \omega_{i} d k_{i}}{(2 \pi)^{2}} \delta\left(\omega_{1}+\ldots \omega_{p}\right) \delta\left(k_{1}+\ldots k_{p}\right) \\
& \times f_{p}\left(r,\left\{k_{j}\right\}\right) G_{p, p}\left(\left\{k_{j}, \omega_{j}, \kappa_{j}, 0\right\}\right) .
\end{aligned}
$$

Here $f_{p}\left(r,\left\{k_{j}\right\}\right)$ are one-dimensional versions of the functions $f_{p}\left(\boldsymbol{r},\left\{\boldsymbol{k}_{j}\right\}\right)$ defined by Eq. (C7). The set $\left\{\kappa_{j}\right\}$ denotes all the outgoing wavevectors.

We consider the skeleton contributions to the nonlinear Green's function $G_{p, p}$, denoted by $G_{p, p}^{\mathrm{s}}$. Similarly to the definition (33) we introduce $T_{p}^{\mathrm{s}}\left(r,\left\{\kappa_{j}\right\}\right)$ as

$$
\begin{aligned}
T_{p}^{\mathrm{s}}\left(r,\left\{k_{j}^{\prime}\right\}\right) & =\int_{-\infty}^{\infty} \prod_{i=1}^{p} \frac{d \omega_{i} d k_{i}}{(2 \pi)^{2}} \delta\left(\omega_{1}+\ldots \omega_{p}\right) \delta\left(k_{1}+\ldots k_{p}\right) \\
& \times f_{p}\left(r,\left\{k_{j}\right\}\right) G_{p, p}^{\mathrm{s}}\left(\left\{k_{j}, \omega_{j}, \kappa_{j}, 0\right\}\right) .
\end{aligned}
$$

Repeating the calculation of Appendix $\mathrm{C}$ in the asymptotic regime $\kappa_{j} r \ll 1$ but with the redefined rung (47) one gets

$$
T_{p}\left(r,\left\{\kappa_{j}\right\}\right)=C_{p}(\bar{\epsilon} r)^{p / 3} r^{p \delta_{\mathrm{a}}} \prod_{j=1}^{p}\left|\kappa_{j}\right|^{1 / 3+\delta_{\mathrm{a}}} .
$$

Here $C_{p}$ are dimensionless constants that absorb all the numerical factors. In fact, the result (51) could be guessed directly by recognizing that every rung which is connected with the "outgoing" Green's functions $G\left(\kappa_{j}, 0\right)$ contributes to $T_{p}\left(r,\left\{\kappa_{j}\right\}\right)$ a factor $\left|\kappa_{j}\right|^{1 / 3+\delta_{a}}$. All together they give $\prod_{j=1}^{p}\left|\kappa_{j}\right|^{1 / 3+\delta_{\mathrm{a}}}$. Convergence of the integrals over $\kappa_{j}$ and $\omega_{j}$ implies that neither inner nor outer scales may appear, and therefore dimensional consideration require a factor $r^{p\left(\frac{1}{3}+\delta_{\mathrm{a}}\right)}$.

\section{The 2nd and 3rd order correlation functions: relations between $\delta_{\mathrm{a}}, \delta_{2}$ and $\tilde{\delta}$}

Consider first the scaling exponent $\zeta_{2}$. In Subsect. $\mathrm{VA}$ we showed that the resummation of the ladder diagrams leads to an anomalous correction to the exponent $\zeta_{2}^{\mathrm{K} 41}=\frac{2}{3}$ which is $-\tilde{\delta}$ (cf. Eq. (46). But according to Eq. (51) the ladder in the skeleton contribution brings in an additional correction $2 \delta_{\mathrm{a}}$. Altogether we have in the 1-loop order

$$
\zeta_{2}=\frac{2}{3}-\tilde{\delta}+2 \delta_{\mathrm{a}} .
$$

Therefore the exponent $\delta_{2}$ defined by (38) may be expressed as follows:

$$
\delta_{2}=2 \delta_{\mathrm{a}}-\tilde{\delta}
$$

Another relation between the exponents will follow from the analysis of the fusion of three points. To 1loop order the nonlinear Green's function $G_{3,3}$ has the skeleton contribution diagram 3 in Fig. 何, and the 1loop diagrams in Fig. $4 \mathrm{~b}$. The skeleton contribution can be read directly from Eq. (51):

$$
T_{3}^{\mathrm{s}}\left(r,\left\{\kappa_{j}\right\}\right)=C_{3} \bar{\epsilon} r r^{3 \delta_{\mathrm{a}}}\left|\kappa_{1} \kappa_{2} \kappa_{3}\right|^{1 / 3+\delta_{\mathrm{a}}} .
$$

To discuss the other contributions we refer to Fig. 1 in which all the diagram of $G_{3,3}$ with zero, one and two rungs are represented. We have one diagram with no rung, three with one, nine with 2 . The multiplicity of 3 in the diagrams of type (2) represent the three possible connections of two struts by two rungs. The multiplicity of 6 in the diagrams of type (3) represent the different pair-permutations of three struts. In general there are $3^{n}$ diagrams with $n$ rungs, out of which three will have one disconnected strut. Diagrams with disconnected struts will not contribute in the asymptotic regime that interests us here. Thus out of the diagrams in Fig. Aa only the skeleton diagram (3) remains in the asymptotic regime.

In general, with $n$ rungs we have $3^{n}-3$ fully linked diagrams. This number is $6\left[\left(3^{n-1}-1\right) / 2\right]$, and the number $\left[\left(3^{n-1}-1\right) / 2\right]$ counts the topologically distinct fully linked diagrams with $n$ rungs. Thus for example we represent in Fig. 4 $4 \mathrm{~b}$ the four topologically distinct contributions with three rungs. These are all the 1-loop ladder diagrams contributing to the 3'rd order correlation function. We show now that of these four terms diagram (1a) does not contribute a logarithmic divergence, whereas the other three contribute the same logarithmic term. In fact, this is the beginning of a systematic rule: the only diagrams that contribute logarithmic terms in the 1-loop order are those in which the last rung appears 
to the right of the skeleton diagrams. Similar rules will be established below for higher loop contributions.

Consider then the 1-loop diagram (1a) in Fig. Ab in which this rule is not obeyed. We focus on the loop made by the two rungs and the Green's functions $q$ and $q^{\prime}$, considering the asymptotic regime $k_{a}, k_{b} \gg q \gg \kappa_{a}, \kappa_{b}$. This is the only regime in which a logarithmic divergence is possible. In this regime $q^{\prime} \approx q^{\prime \prime} \approx k_{a}+k_{b}$. Thus the rung $R_{a}\left(k_{a}, k_{b}, q, q^{\prime}\right)$ contributes to the loop $q^{1 / 3+\delta_{a}}$. The rung $R_{q, q^{\prime}, \kappa_{a}, q^{\prime \prime}}$ and the Green's function $G\left(q^{\prime}\right)$ do not contribute any $q$ dependence to the integrand. The $\omega$ integration over the product of the Green's functions $G(q)$ and $G\left(q^{\prime}\right)$ gives approximately $1 / \gamma\left(q^{\prime}\right)$ and again contributes no $q$ dependence. Finally we have the evaluation

$$
T_{3}^{(1 a)} \propto \int_{\kappa}^{k_{a}} d q q^{1 / 3+\delta_{a}} .
$$

Clearly, this diagram does not exhibit a logarithmic divergence and as such it does not contribute to the renormalization of the scaling exponent.

The other three diagrams in Fig. Th (namely 1b,1c and 1d) are different, they all have a logarithmic divergence. The reason for the difference is that in these three diagrams there are four Green's functions, instead of five in diagram (1a), which carry large wavevectors. This is the same situation as in the skeleton diagram (3) in Fig. 27a. In the loop we have now two Green's functions, instead of one in diagram (1a), that carry small wavevectors $q$. This difference leads to a different $q$ dependence in the loop, and to a logarithmic divergence. We demonstrate this explicitly in the next paragraph, but we already draw the conclusion which is general: 1-loop ladder diagrams with logarithmic divergences are those in which the additional rung (compared to skeleton diagram) has been positioned to the right of the skeleton structure.

Explicitly, consider diagram (1b) in Fig. 凷. The rung $R_{a}\left(k_{a}, k_{b}, q, q^{\prime}\right)$ contributes $q^{1 / 3+\delta_{a}}$ as before. But now also the rung $R_{a}\left(q^{\prime}, k_{c},-q, \kappa_{c}\right)$ contributes the same $q$-dependence. On the other hand the rung $R_{a}\left(q,-q, \kappa_{a}, \kappa_{b}\right)$ contributes $|q|^{-1-2 \delta_{a}}$. The $\omega$ integration with the product of the two Green's functions $G(q, \omega) G(-q,-\omega)$ is the same as (33) leading to $-1 / 2 \gamma(q)$. In total we have a logarithmic integral.

The diagram (1d) is very similar to (1b); it has the same rung structure at the left, and the rightmost rung is $R_{a}\left(q,-q, \kappa_{a}, \kappa_{c}\right)$. This makes no difference to the $q$ dependence and thus to the logarithmic divergence or to the factor in front of the logarithm. Diagram (1c) is slightly different, having the third rung on the same ladder as the second rung. Nevertheless the rung $R_{a}\left(q^{\prime}, k_{c}, q,-q\right)$ ) contributes exactly the same $q$ dependence as the two rungs in diagrams (1b) or (1d). Thus it yields at the end the same factor with the same logarithm. Finally, comparing 1c to diagram 3 in Fig. 2a we see that the loop structures are identical in both, and thus if diagram
3 had a prefactor $\tilde{\delta}$, we can immediately conclude that the three diagrams (1b, 1c and $1 \mathrm{~d})$ will result in a total prefactor of $3 \tilde{\delta}$ :

$$
T_{3}^{(1)}\left(r,\left\{\kappa_{j}\right\}\right)=3 \tilde{\delta} \ln \left[\frac{1}{r \kappa}\right] T_{3}^{(s)}\left(r,\left\{\kappa_{j}\right\}\right),
$$

where $\kappa \equiv\left[\kappa_{1} \kappa_{2} \kappa_{3}\right]^{1 / 3}$. The leading contribution from the higher loop diagrams can be seen to contribute higher order terms in the series of a power law, similarly to the mechanism displayed in Eqs. 43 46):

$$
T_{3}\left(r,\left\{\kappa_{j}\right\}\right)=\frac{T_{3}^{(s)}\left(r,\left\{\kappa_{j}\right\}\right)}{[r \kappa]^{3 \tilde{\delta}}} .
$$

Substituting (54) we find finally

$$
T_{3}\left(r,\left\{\kappa_{j}\right\}\right)=C_{3} \bar{\epsilon}[r \kappa]^{1+3 \delta_{a}-3 \tilde{\delta}} .
$$

Accordingly to 1-loop order we write

$$
\zeta_{3}=1+3 \delta_{a}-3 \tilde{\delta} .
$$

At this point we use the exact, nonperturbative result that $\zeta_{3}=1$ to find the relationship between $\delta_{a}$ and $\tilde{\delta}: \quad \delta_{a}=\tilde{\delta}$. Together with 53 we get the important conclusion that all our $\delta$ 's are the same:

$$
\delta_{2}=\delta_{a}=\tilde{\delta}=\zeta_{2}-\zeta_{2}^{\mathrm{K} 41} \approx 0.03 .
$$

We should stress that this important result is obtained using only the asymptotic scaling properties of the rung. Changing the explicit form of the rung without ruining the asymptotics will affect only the subleading terms in the analysis. The leading logarithmic terms are insensitive to the details of the analytic form of the rung.

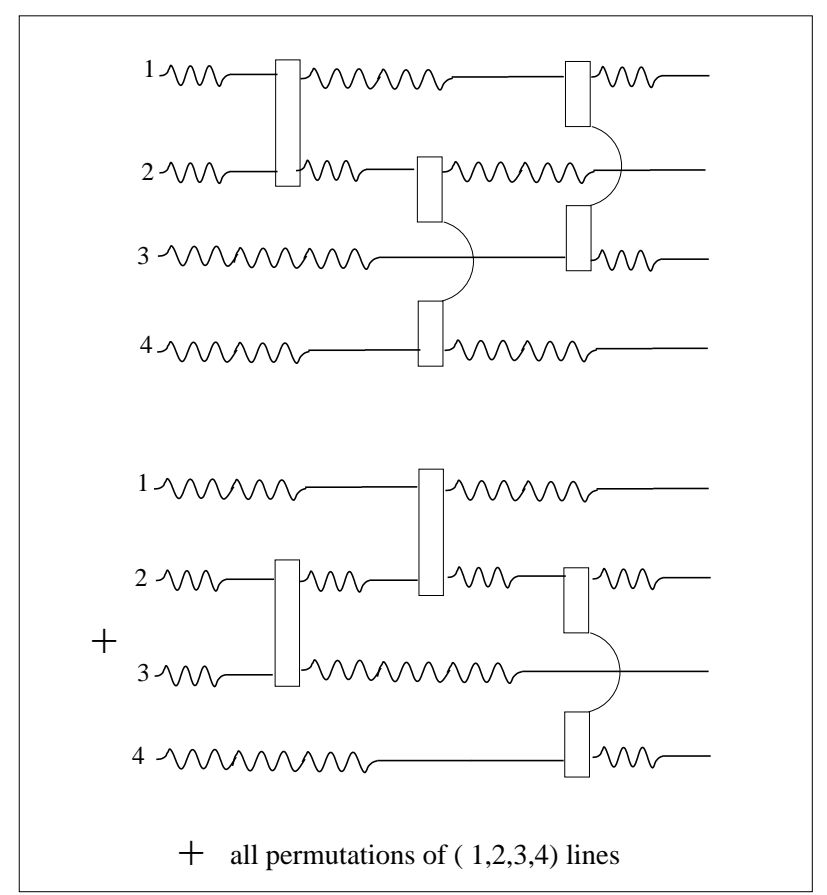

FIG. 5. The skeleton contributions in the diagrammatic expansion of $\mathbf{G}_{4,4}$. 


\section{ANOMALOUS SCALING EXPONENTS IN THE 1-LOOP APPROXIMATION: SURPRISE, SURPRISE}

We are poised to compute now the anomalous corrections to all the scaling exponents of the $p$-order correlation functions in the 1-loop approximation. Start with the 4th order nonlinear Green's function, and consider the skeleton diagrams in Fig. 5. In the one loop order, to obtain a logarithmic divergence in the asymptotic regime we must add the additional rung on the right of the skeleton structure. The combinatorics are elementary: Each skeleton diagram can host a new rung on the right in six different ways. Once a rung has been put in place the leading (logarithmic) contribution to the loop integral is the same as the loop integrals considered in the last Section. It gives the same logarithm with the same prefactor. The only difference is in the combinatorics. We can thus write by inspection

$$
T_{4}^{(1)}\left(r,\left\{\kappa_{j}\right\}\right)=6 \tilde{\delta} \ln \left[\frac{1}{r \kappa}\right] T_{4}^{(s)}\left(r,\left\{\kappa_{j}\right\}\right),
$$

where $\kappa$ is the geometric mean of all the $\kappa_{j}$. Resumming the leading contributions of the higher order loop diagrams results in the power law

$$
T_{4}\left(r,\left\{\kappa_{j}\right\}\right)=\frac{T_{4}^{(s)}\left(r,\left\{\kappa_{j}\right\}\right)}{[r \kappa]^{6 \tilde{\delta}}} .
$$

Using Eq. (51) we reach the final result

$$
\zeta_{4}=4 / 3+4 \delta_{a}-6 \tilde{\delta}=4 / 3-2 \delta_{2}, \quad \text { 1-loop order. }
$$

The analysis of the 1-loop order contribution to the anomalous exponents of the $p$-order correlation functions is as straightforward. There are $p(p-1) / 2$ possibilities to append an additional rung to the right of the skeleton structure of the $p$-order nonlinear Green's function. All these diagrams contribute identical leading order logarithmic terms, with the same prefactor, summing up to an anomalous correction to the scaling exponent of the skeleton diagrams which is $\tilde{\delta} p(p-1) / 2$. According to Eq. (51) the scaling exponent of the skeleton contribution itself is corrected with respect to K41 by $p \delta_{a}$. Thus altogether

$$
\zeta_{p}=\frac{p}{3}+p \delta_{a}-\tilde{\delta} \frac{p(p-1)}{2} .
$$

Using Eq. (60)

$$
\zeta_{p}=\frac{p}{3}-\delta_{2} \frac{p(p-3)}{2}, \quad \text { 1-loop order. }
$$

We note that this formula, which is valid in our case to 1-loop order only, is identical in prediction to Kolmogorov's log-normal phenomenological model (known as K62). This is interesting, as it stems from the nontrivial topology of the ladder diagrams, in which only the most leading were considered. The present authors find the connection between lognormality and ladder diagrams unexpected.

Nevertheless we should recognize that in the present approach this result has a limited region of validity. The analysis of the 2-loop order which is provided below will show that Eq. (4) is only valid when $p \delta_{2} \ll 1$. The 2 loop order will contribute positive terms of the order of $\delta_{2}^{2} p^{2}(p-3)$, reducing the negative tendency of the correction to K41. Accordingly the present theory will not suffer from the well know deficiencies of the K62 log-normal model which for us is only a first order result.

\section{ANOMALOUS SCALING EXPONENTS IN THE 2-LOOP APPROXIMATION: K62 IS CURED}

In this Section we calculate the 2-loop contributions to the scaling exponents $\zeta_{p}$. Even though these contributions are very small when $p \delta_{2}$ is small (for, say, $p<6$ ), they become important for larger values of $p$ where K62 begins to turn down the $n$ dependence of $\zeta_{n}$. In addition this calculation allows to present clear ranges of validity for the 1-loop and 2-loop calculations.

\section{A. 2-loop contributions to $\zeta_{2}$}

We consider the 2-loop diagram (4) in Fig. 2a. Substituting it instead of $G_{2,2}$ in Eq. (39) we obtain the quantity $T_{2}^{(2)}(r, \kappa)$. We want to compute the correction that this diagram gives to the skeleton diagram (2), and to this aim we divide it by $T_{2}^{\mathrm{s}}(r, \kappa)$. In the asymptotic regime $\kappa r \ll 1$ the loop integrals over $q_{1}$ and $q_{2}$ contribute mostly in the range $k \gg q_{1}, q_{2} \gg \kappa$. In this regime the integrals over $k_{a}, \omega_{a}$ cancel from the ratio of $T_{2}^{(2)}(r, \kappa) / T_{2}^{\mathrm{s}}(r, \kappa)$. In addition the Green's functions $G\left(\kappa_{c}\right)$ and $G\left(\kappa_{d}\right)$ cancel. Thus this ratio can be read from the ratio of the corresponding diagrams for $G_{2,2}$, or taking the diagram (4) and amputating the incoming and outgoing legs. We still need to divide by the rung in diagram (2) where $k_{a}$ is replaced by $1 / r$ :

$$
\begin{aligned}
& \frac{T_{2}^{(2)}(r, \kappa)}{T_{2}^{\mathrm{s}}(r, \kappa)}=\frac{1}{R(1 / r,-1 / r, \kappa,-\kappa)} \int_{-\infty}^{\infty} \frac{d q_{1} d q_{2} J\left(q_{1}\right) J\left(q_{2}\right)}{(2 \pi) q_{1} q_{2}} \\
& \times R\left(r^{-1},-r^{-1}, q_{1},-q_{1}\right) R\left(q_{1},-q_{1}, q_{2},-q_{2}\right) R\left(q_{2},-q_{2}, \kappa,-\kappa\right), \\
& J(q)=\int_{-\infty}^{\infty} \frac{d \omega}{2 \pi} G(q, \omega) G(-q,-\omega)=-\frac{1}{2 \gamma(q)} .
\end{aligned}
$$

In Appendix D we analyze this integral in the asymptotic limit $\kappa r \ll 1$ with the final result 


$$
T_{2}^{(2)}(r, \kappa)=\tilde{\delta}^{2}\left[\frac{1}{2} \ln ^{2}\left(\frac{1}{\kappa r}\right)+b_{1} \ln \left(\frac{1}{\kappa r}\right)\right] T_{2}^{\mathrm{s}}(r, \kappa),
$$

where $b_{1}$ is a dimensionless constant

$$
b_{1} \approx-0.434 \text {. }
$$

In Eq. (67) the $\ln ^{2}$ term accounts for the exponentiation of the 1-loop contribution, whereas the $\ln$ term provides the 2-loop correction to the scaling exponent $\zeta_{2}$. Instead of Eq. (53) we now read

$$
\delta_{2}=2 \delta_{a}-\tilde{\delta}-b_{1} \tilde{\delta}^{2} .
$$

A second relation between these exponents will be derived in the next subsection.

\section{B. 2-loop contributions to $\zeta_{3}$}

The calculation to $O\left(\delta_{2}^{2}\right)$ of the contributions to $\zeta_{3}$ and of higher order $\zeta_{p}$ due to ladder resummations introduces for the first time 6-point irreducible interactions amplitudes. These appear in the ladder diagrams as rungs with six legs, arising from diagrams that due to their topology cannot be resummed into reducible contributions consisting of two 4-point rungs and one Green's function. The 6-point rung is discussed in Appendices A and G. In particular in Appendix $\mathrm{G}$ we explain why the functional dependence of $\zeta_{p}$ on $p$ can be understood completely on the basis of the analysis of ladders with 4-point rungs. This stems from the fact that the reducible and irreducible contributions to the 6-point rung are of the same order, and their combinatorical factors are identical.

There are many possible two loop diagrams involving 4-point rungs that appear in the expansion of $G_{3,3}$. However, we are only interested in those contributing a logarithmic divergence in the asymptotic regime. As before, to get the relevant diagrams we need to append the last rung to the right of the existing 1-loop structure. Thus, we begin with the 3 logarithmic diagrams in Fig. 四 (i.e. $1 \mathrm{~b}, 1 \mathrm{c}$ and $1 \mathrm{~d}$ ) and consider all the diagrams that are obtained by adding an additional rung on the right which connects two struts. The nine resulting diagrams are shown in Appendix E. These diagrams are subdivided into two groups: three diagrams in which the last rung connects the same struts as the previous rung, and six diagrams in which the last rung connects different struts. In Appendix $\mathbf{F}$ we explain that the first group of diagrams gives exactly the same asymptotic integral as the 2-loop contribution to $\zeta_{2}$. This statement should be reiterated because of its importance to the structure of the theory: the integrals are different, but once the limits $k \gg q_{1}, q_{2} \gg \kappa$ are taken, the resulting integrals coalesce with those computed in the previous Subsection. Thus the contribution to $\zeta_{3}$ from these three diagrams will be
$3 b_{1} \tilde{\delta}^{2}$ (which is 3 times larger than the corresponding contribution to $\left.\zeta_{2}\right)$.

The six diagrams of the second group look topologically different, but again in the asymptotic regime coalesce into an identical integral Eq. (E1) with $\tilde{\Psi} \rightarrow \tilde{\Psi}_{2}$, where

$$
=\frac{\tilde{\Psi}_{2}\left(q_{1}, q_{2}\right)}{\left(\left|q_{1}\right|^{2 / 3}+\left|q_{2}\right|^{2 / 3}+\left|q_{1}+q_{2}\right|^{2 / 3}\right)\left(q_{1}^{2}+q_{2} q_{1}+q_{2}^{2}\right)} .
$$

Following the procedure outlined in Appendix $\mathrm{E}$ we find the coefficients of expansion

$$
a_{2}=1, \quad b_{2} \approx-0.55 .
$$

Finally we get the 2-loop form of $\zeta_{3}$ :

$$
\zeta_{3}=1+3 \delta_{a}-3 \tilde{\delta}-\tilde{\delta}^{2}\left(3 b_{1}+6 b_{2}\right) .
$$

Demanding again $\zeta_{3}=1$ we find from Eqs. (69, 72):

$$
\begin{aligned}
\tilde{\delta} & =\delta_{2}-\left(b_{1}+4 b_{2}\right) \delta_{2}^{2}+O\left(\delta_{2}^{3}\right), \\
\delta_{a} & =\tilde{\delta}\left[1+\left(b_{1}+2 b_{2}\right) \tilde{\delta}\right]
\end{aligned}
$$

These results are used in the next Subsection to calculate $\zeta_{n}$ for $n \geq 3$ to 2-loop order.

\section{2-loop contributions to $\zeta_{p}, p \geq 4$}

The calculation of the contribution of 4-point rungs to $\zeta_{p}$ for higher values of $p$ does not necessitate the evaluation of new integrals. In Appendix $\mathbf{E}$ we explain that all the 2-loop integrals appearing in the ladders of $G_{4,4}$ and higher order nonlinear Green's functions are identical in the asymptotic regime to one of the two integrals appearing in the 3-order quantity. The only differences are in the combinatorial factors that account for how many ways we can choose the rungs to connect between $p$ struts.

If the second rung is connecting the same struts as the rung before it we have the same combinatorial factor as in the 1-loop order, namely $p(p-1) / 2$. This provides a contribution to $\zeta_{p}$ which is $p(p-1) \delta^{2} b_{1} / 2$. If the second rung is not connecting the same struts as the rung before it, we have $p(p-1)(p-2)$ contributions. This is due to the existence of $p(p-1) / 2$ ways connect two struts with the first rung, and then $2(p-2)$ ways to connect one of these two struts with the remaining $(p-2)$ struts. This leads to a contribution $p(p-1)(p-2) b_{2} \tilde{\delta}^{2}$. We should stress that the loops must have a joint strut to give a ln contribution. Two disconnected loops lead only to $\ln ^{2}$ contributions, which do not enter the 2-loop corrections to the scaling exponents. In total we find 


$$
\begin{aligned}
\zeta_{p} & =\frac{n}{3}+p \delta_{a}-\frac{p(p-1)}{2}\left[\tilde{\delta}+b_{1} \tilde{\delta}^{2}\right] \\
& -p(p-1)(p-2) b_{2} \tilde{\delta}^{2}+O\left(\tilde{\delta}^{3}\right) .
\end{aligned}
$$

Substituting Eqs. (73, 74) we obtain finally

$$
\zeta_{p}=\frac{p}{3}-\frac{p(p-3)}{2} \delta_{2}\left[1+2 \delta_{2} b_{2}(p-2)\right]+O\left(\delta_{2}^{3}\right)
$$

We should stress that the functional form presented in this equation is solid. It is shown in Appendix $\mathrm{G}$ that the contribution coming from 6-point irreducible rungs is only renormalizing the value of $b_{2}$ which anyway depends on the precise analytic form of the 4-point rung which is not available at the present time. We estimate the range of validity of this order of the calculation by the Hölder inequalities, which disallow a nonlinear increase in the $\zeta_{p}$ as a function of $p$. The inflection point where this requirement is violated may serve as a good estimate for the range of validity. This inflection point occurs at $p \approx 1.4-1 /\left(6 b_{2} \delta_{2}\right) \approx 12$. In Fig. 1 1 we show, within this range, the K41 prediction, the 1-loop approximation (equivalent to K62) and our 2-loop final result. It is obvious that the 2-loop loop prediction goes considerably beyond the range of validity of the K62 formula which has an unphysical maximum at $p \approx 11.5$. We believe that all the reliably measured values of $\zeta_{p}$ agree very well with this prediction.

Using the bridge relation [6] $\mu=2-\zeta_{6}$ we predict

$$
\mu=9 \delta_{2}\left(1+8 b_{2} \delta_{2}\right) .
$$

Plugging in the numbers we get $\mu=0.235+O\left(\delta_{2}^{3}\right)$. This is to be contrasted with the K62 prediction $\mu \approx 0.27$. We conclude that the 2-loop contribution is very significant for experimentally measured exponents. If one wishes to obtain theoretical results for $\zeta_{p}$ with higher values of $p$ one needs to consider the 3-loop contributions, which pose no further conceptual difficulties. Nevertheless the experimental situation does not warrant at the present time the effort needed to accomplish such a calculation.

One should stress before closing this Section that the form of Eq. (76) is universal, stemming from the structure of the ladder diagrams and from combinatorics only. However the numerical value of $b_{2}$ is model dependent. We have checked that changing the form of the rung keeping the asymptotics unchanged results in $b_{2}$ remaining negative while its value not changing by more than a factor of 2 or so. At this moment in time one can determine $b_{2}$ using the value of $\zeta_{4}$ from experiments, allowing us then to predict accurate values of $\zeta_{n}$ for $n$ up to 12 . It is our plan however to develop in the near future a theoretical equations for the 4-point and 6-point rungs, leading to an ab-inito determination of their analytic forms, and with them of the parameters $\tilde{\delta}$ and $b_{2}$.

\section{SUMMARY AND DISCUSSION}

The main steps of this and previous papers leading to the present results have been as follows:

- The theory is developed using BL-velocities to eliminate the spurious infrared divergences that are due to sweeping effects when Eulerian velocities are employed.

- The Dyson-Wyld perturbation theory was line resummed in order to achieve order by order convergent perturbation theory with K41 propagators as the lines in the theory. At this point the objects of the theory are two 2-point propagators (Green's function and correlator) and one 3-point vertex. The 3-point vertex is in no way "small", and renormalizing it does not change this fact [24].

- Multipoint correlation functions are considered when $p$ coordinates coalesce together. In the fusion limit $\kappa r \rightarrow 0$ it is advantageous to reorganize the theory in terms of one propagator (K41 Green's function), and 4-point, 6-point vertices etc. (the rungs). The series of diagrams contributing to the fusion limit are then simple ladder diagrams.

- The crucial step of the theory is achieved by two requirements: (i) the 4-point rung should be consistent at the level of the skeleton diagrams with the fusion rules with K41 scaling exponents. (ii) The resummation of the ladder diagrams that appear when 2 coordinates fuse together should lead to the correct value of $\zeta_{2}$. These double requirements accomplish two things in one go: (i) the theory is now developed around the K41 limit, leading to the appearance of the small parameter $\delta_{2}$ in front of the 4-point rung, and (ii) all the anomalies are coming from the ladder resummations. The 6-point rung is shown explicitly (Appendix G) to be of second order in the small parameter, 8-point rungs are of third order, etc.

- We computed the anomalous exponents in 1-loop order, inputting the value of $\zeta_{2}$ and requiring that $\zeta_{3}=1$. The result is that the scaling exponents are predicted to this order to agree with the log normal model K62. We showed that to this order the result is universal, independent of the simplifications and of the model form of the rung.

- We computed the anomalous exponents in 2-loop order. The malaise of K62 is cured, the 2-loop contribution has a sign that lifts up the exponents from the down curve of the K62 parabola. While the form of the 2-loop result is universal, the numerical value of the parameter $b_{2}$ appearing in the final result is model dependent, with contributions for the 4 -point and 6-point rungs. 
To improve upon the present theory one needs to develop a theory for the 4-point and 6-point interaction amplitudes. Here we determined only the asymptotic properties of the 4-point rung, and this allowed us to predict the form of the scaling exponents, but an input of the value of the anomalous part of $\zeta_{2}$ was needed to nail the 1-loop order. In fact we could use the value of $\zeta_{4}$ to fix the value of $b_{2}$ and gain a solid prediction of all the exponents to 2-loop order. Such a prediction for $\zeta_{n}$ would be valid up to $n \approx 12$. It is very easy to generalize the result that we have to 3 -loop order, with the introduction of yet one more parameter associated with the 3-loop integrals, say $b_{3}$, which included also contributions from the irreducible 8-point rung. The result would read

$$
\begin{aligned}
\zeta_{n} & =\frac{n}{3}-\frac{n(n-3)}{2} \delta_{2}\left[1+2 \delta_{2}(n-2) b_{2}\right. \\
& \left.+6 \delta_{2}^{2} b_{3}(n-1)(n-2)\right]+O\left(\delta_{2}^{4}\right) .
\end{aligned}
$$

We stress that this form stems from the structure of the ladder diagrams, and we consider it very solid. From one point of view we can now use the value of $\zeta_{5}$ to fix $b_{3}$ to provide a prediction that is valid for any $n$ within experimental reach for quite some time. But this is not the main point. The main point is that we have identified the coefficients appearing in this formula with particular objects, i.e the 4-point and higher order vertices which appear in the theory as the rungs of the ladders. Obviously, a calculation of the renormalized rungs from first principle would remove the need to input experimental information altogether, affording us a complete theory of the scaling exponents of isotropic turbulence. At this point this is still not in the cards.

\section{ACKNOWLEDGMENTS}

It is a pleasure to thank Anna Pomyalov for her patient help with the diagrams in this paper. We thank her, Yoram Cohen, Ayse Erzan and Massimo Vergassola for useful comments on the manuscript. This work has been supported in part by the Israel Science Foundation, the German-Isreali Foundation, the European Commission under contract HPRN-CT-2000-00162 ("Nonideal Turbulence"), and the Naftali and Anna BackenrothBronicki Fund for Research in Chaos and Complexity.

\section{APPENDIX A: EXPLANATION OF THE DIAGRAMMATIC EXPANSION IN FIGS. 2, 3}

It is important to stress that in the present theory we take into account all the necessary contributions. To understand this we need to say a few more words about the representation of $G_{2,2}$ in terms of simple ladders only, and of the $n$th order correlation function with $p$ fused coordinates as shown in Fig. 3.
The natural objects in the straightforward perturbation theory are the 2-point correlation function and Green's function (11), and the 3-point vertex resulting from the Navier-Stokes nonlinearity $\boldsymbol{u} \cdot \boldsymbol{\nabla} \boldsymbol{u}$. After line resummation the theory contains "dressed" correlator and Green's function. The 3-point vertex is protected by Galilean invariance and is not effected much by dressing [24]. Thus, when we write the expansion of $G_{2,2}$ many diagrams involving these objects appear. The strategy that leads to the simple ladder expansion of Fig. 2 is as follows: Every diagram that contributes to the series is inspected for its cross section, or in other words what are the kind of objects that intersect a line cutting across the diagram. The line is put at the left of the diagram, and is moved to the right. Every time that the line intersects two Green's functions that are oriented as shown in Fig. 2 we mark that position, and move the line further to the right, until we intersect again two Green's functions, etc. For every pair of such intersections we now sum up all the topologically allowed diagrams that can be inflated ad infinitum from the fragments appearing between the two pairs of Green's functions. This infinite resummation is the representation of the rung, which is actually a 4-point vertex. This procedure is flawless, taking into account all the possible diagram in the series of $G_{2,2}$ except for one subseries. This is the subseries of diagrams in which the cross section contains exactly two 2-point correlation functions. It was shown in Paper II that this can happen only once per diagram, and therefore we cannot resum such contributions to the rung, since this will lead to one rung differing from all the others. Thus the series shown in Fig. 2a contains in the $+\ldots$ also ladders in which the struts contain two correlators above each other. With these we account for all the possible diagrams in $G_{2,2}$.

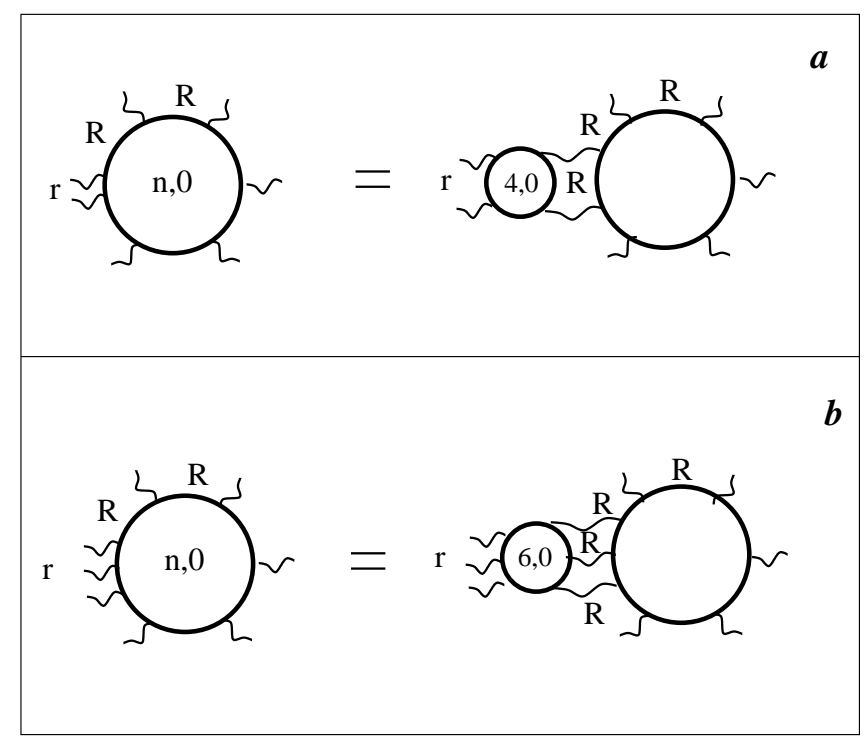


FIG. 6. The contributions to the fusion of 2 and 3 coordinates from diagrams containing two and three correlators in the cross section.

The presence of these diagrams also complicates the discussion of the fusion rules and Fig. 3. When we pool out the fragment containing the two coalescing coordinates we will generate in the series expansion of this fragment also the diagrams containing two correlators in the cross section. To understand their roles we will always consider the fragment of the diagrams to the right of this special cross section as belonging to the main body, see Fig. 6. But now the fragment pulled out becomes a 4 th order correlation function instead of $G_{2,2}$. One can analyze the role of this diagram in the fusion limit, and the conclusion is that when $r \ll R$ it contributes the same power $(R / r)^{\zeta_{2}}$ as the diagrams considered in the body of the paper. We thus conclude that the procedure followed in the body of the paper is amply sufficient for the calculation of $\zeta_{2}$.

The same type of considerations apply when we fuse $p$ coordinates. In the series expansion for $G_{p, p}$ we will have however ladder diagrams whose topology requires resummation into $2 p$-point irreducible interaction amplitudes which serve as new types of rungs. The first one appears at the level of $G_{3,3}$, and is the 6-point rung that is discussed explicitly in Appendix G. It will be argued that the 6-point rung is of second order in the smallness $\delta_{2}$ that characterizes the 4-point rung. Respectively the $2 p$-point rungs are of $O\left(\delta_{2}^{p-1}\right)$ and thus rungs with $p>3$ will not affect the analysis of this paper.

On top of the diagrams considered in the body of the paper one needs to consider those having $p$ correlators in the cross section. The conclusion is however the same the diagrams considered give all the necessary information, no new (or more divergent) information is available in the diagrams that we do not consider explicitly.

At the end of the day the procedure of calculating the scaling exponents to $O\left(\delta_{2}^{2}\right)$ leaves us with only three dressed objects in the theory, the dressed 2-point Greens' function and new 4-point and 6-point dressed vertices which we call the rungs. We will show that the rungs are small and therefore these three objects suffice for a consistent and controlled theory of the scaling exponents.

A reader who is an expert in diagrammatic theories may feel worried that the procedure advocated here mixes into the 4-point vertex some 2-particle reducible diagrams. This is indeed so. In other words (and see Paper III for more details) there are contributions in which the cross section includes two propagators, like one Green's function and one correlator, or two Green's functions oriented oppositely to the ones plotted in Figs. 2 and 3, which we resum into our 4-point vertex. Usually one would prefer not to include 2-particle reducible diagrams in the 4-point vertex, but rather to distinguish different 4-point vertices. In our case one would need three different 4-point rungs, all presented in detail in Paper III. Indeed, if we planned to compute our 4-point vertices from summing up diagrams it would be very advisable to distinguish different types of rungs. In this paper we determine however the properties of the 4-point vertex from the fusion rules, and it makes no difference at all how we classify the diagrams. For the sake of clear presentation it is much better to have just one type of rung. This rung is 2-particle reducible and hides three types of 2-particle irreducible rungs as a single object. The conclusions of the analysis are independent of this simplification.

\section{APPENDIX B: RESUMMATION INTO DIAGONAL K41 PROPAGATORS}

The starting point of this rearrangement are the mass operators in $k, \omega$ representation $\Sigma_{\alpha \beta}\left(\boldsymbol{r}_{0} \mid \boldsymbol{k}_{1}, \boldsymbol{k}_{2}, \omega\right)$ and $\Phi_{\alpha \beta}\left(\boldsymbol{r}_{0} \mid \boldsymbol{k}_{1}, \boldsymbol{k}_{2}, \omega\right)$. Define the "diagonal" part of the mass operators as

$$
\begin{aligned}
\sigma_{\alpha \beta}\left(\frac{\boldsymbol{k}_{1}+\boldsymbol{k}_{2}}{2}\right) & \equiv \int \frac{d\left(\boldsymbol{k}_{1}-\boldsymbol{k}_{2}\right)}{(2 \pi)^{3}} \Sigma_{\alpha \beta}\left(\boldsymbol{k}_{1}, \boldsymbol{k}_{2}, 0\right), \\
\phi_{\alpha \beta}\left(\frac{\boldsymbol{k}_{1}+\boldsymbol{k}_{2}}{2}\right) & \equiv \int \frac{d\left(\boldsymbol{k}_{1}-\boldsymbol{k}_{2}\right)}{(2 \pi)^{3}} \Phi_{\alpha \beta}\left(\boldsymbol{k}_{1}, \boldsymbol{k}_{2}, 0\right) .
\end{aligned}
$$

In these definitions $\boldsymbol{r}_{0}$ disappears. The reason is that for objects which are time independent the Eulerian and BL-representations are equivalent and the designation $\boldsymbol{r}_{0}$ is unneeded. Here we have objects with $\omega=0$, or timeintegrated quantities. It was shown in ref. 25] that time integrated quantities are related to simultaneous correlations, and as such they lose the $\boldsymbol{r}_{0}$ designation.

Denote the rest of the mass operators as

$$
\begin{aligned}
& \tilde{\Sigma}_{\alpha \beta}\left(\boldsymbol{r}_{0} \mid \boldsymbol{k}_{1}, \boldsymbol{k}_{2}, \omega\right) \equiv \Sigma_{\alpha \beta}\left(\boldsymbol{r}_{0} \mid \boldsymbol{k}_{1}, \boldsymbol{k}_{2}, \omega\right) \sigma_{\alpha \beta}\left(\frac{\boldsymbol{k}_{1}+\boldsymbol{k}_{2}}{2}\right), \\
& \tilde{\Phi}_{\alpha \beta}\left(\boldsymbol{r}_{0} \mid \boldsymbol{k}_{1}, \boldsymbol{k}_{2}, \omega\right) \equiv \Phi_{\alpha \beta}\left(\boldsymbol{r}_{0} \mid \boldsymbol{k}_{1}, \boldsymbol{k}_{2}, \omega\right)-\phi_{\alpha \beta}\left(\frac{\boldsymbol{k}_{1}+\boldsymbol{k}_{2}}{2}\right) .
\end{aligned}
$$

For translationally invariant tensors in homogeneous and incompressible turbulence one can write:

$$
\begin{aligned}
\sigma_{\alpha \beta}(\boldsymbol{k}) & =P_{\alpha \beta}(\boldsymbol{k}) \sigma(k), \\
\phi_{\alpha \beta}(\boldsymbol{k}) & =P_{\alpha \beta}(\boldsymbol{k}) \phi(k),
\end{aligned}
$$

where $P_{\alpha \beta}(\boldsymbol{k})$ is the transverse projector,

$$
P_{\alpha \beta}(\boldsymbol{k})=\delta_{\alpha \beta}-\frac{k_{\alpha} k_{\beta}}{k^{2}} .
$$

It is known [19] that $\sigma(k)$ (which is the mass operator taken at $\omega=0)$ is purely imaginary

$$
\sigma(k)=-i \gamma(k),
$$

with $\gamma(k)$ real positive. On the other hand $\phi(k)$ is purely real. The diagrammatic series expansion of both $\gamma(k)$ 
and $\phi(k)$ converge order by order, and using scaling relations as shown in (16) one can find their scaling behavior. The order-by-order theory dictates a K41 evaluation of these objects which is

$$
\begin{aligned}
& \gamma(k)=c_{\gamma}[\bar{\epsilon} k]^{2 / 3} \\
& \phi(k)=c_{\phi} \bar{\epsilon} k^{-3}
\end{aligned}
$$

where $c_{\gamma}$ and $c_{\phi}$ are dimensionless constants.

The Dyson-Wyld equations can be written shortly as

$$
\begin{aligned}
& \left(\omega+i \nu k^{2}\right) \boldsymbol{G}=\boldsymbol{P}+\boldsymbol{\Sigma} * \boldsymbol{G}, \\
& \boldsymbol{F}=\boldsymbol{G} *(\boldsymbol{\Phi}+\boldsymbol{D}) * \boldsymbol{G}
\end{aligned}
$$

where $\nu$ is the molecular viscosity, $\boldsymbol{P}$ is the transverse projector, and $\boldsymbol{D}$ is the correlation function of the external force which is localized in the energy containing interval. The symbol $*$ stands for summation over tensor indices and integration over intermediate $\boldsymbol{k}$. Substituting $\boldsymbol{\Sigma}$ from Eq. (B3) into the Dyson equation we rewrite:

$$
\left[\omega+i \nu k^{2}+i \gamma(k)\right] \boldsymbol{G}=\boldsymbol{P}+\tilde{\boldsymbol{\Sigma}} * \boldsymbol{G}
$$

In the bulk of the inertial interval we can neglect $\nu k^{2}$ with impunity. The zero order solution of this equation is obtained by neglecting $\tilde{\boldsymbol{\Sigma}}$ :

$$
\begin{aligned}
G_{\alpha \beta} \rightarrow g_{\alpha \beta}(\boldsymbol{k}, \omega) & =P_{\alpha \beta}(\boldsymbol{k}) g(k, \omega), \\
g(k, \omega) & =\frac{1}{\omega+i \gamma(k)} .
\end{aligned}
$$

The zero order solution of $\boldsymbol{F}$ is obtained in three steps: first replace $\boldsymbol{\Phi}$ by $\phi$, secondly neglect $\boldsymbol{D}$ in the inertial interval in comparison with $\boldsymbol{\phi}$, and lastly substitute $\boldsymbol{g}$ instead of $\boldsymbol{G}$ in Eq. (B9). The result is

$$
\begin{aligned}
F_{\alpha \beta} \rightarrow f_{\alpha \beta}(\boldsymbol{k}, \omega) & =P_{\alpha \beta}(\boldsymbol{k}) f(k, \omega) \\
f(k, \omega) & =\frac{\phi(k)}{\omega^{2}+\gamma^{2}(k)} .
\end{aligned}
$$

Iterating Eqs. (B8, B9) without the bare forcing and viscosity results in a new diagrammatic series, which topologically is exactly the same as the old Wyld diagrammatic expansion before line re-summation. The difference is twofold. First, instead of bare propagators we have K41 propagators $\boldsymbol{g}$ and $\boldsymbol{f}$, and every 1-particle reducible fragment of any diagram will have a counter term which subtracts its "diagonal" part. This counter term is of no consequence for our procedure here since the diagrams involving it are resummed in the 4-point vertices (the rungs) together with all the other contributions as explained in Appendix A. The resulting topological structure of the ladder diagrams is thus unchanged in the new formulation.

\section{APPENDIX C: SELF CONSISTENCY AT THE LEVEL OF K41}

Before establishing this self consistency we need to pass from correlation functions in $\boldsymbol{k}, \omega$ representation to structure functions. The theory is done naturally in $\boldsymbol{k}, \boldsymbol{\omega}$ representation but the experimental scaling exponents are measured in simultaneous structure functions. We first transform from $\omega$-representation of $p^{\text {th }}$-order correlation function $\mathcal{F}_{p}\left(\left\{\boldsymbol{k}_{j}, \omega_{j}\right\}\right)$ to simultaneous correlation function $\boldsymbol{F}_{p}\left(\left\{\boldsymbol{k}_{j}\right\}\right)$ by the integration:

$$
\boldsymbol{F}_{p}\left(\left\{\boldsymbol{k}_{j}\right\}\right)=\int_{-\infty}^{\infty} \prod_{i=1}^{p} \frac{d \omega_{i}}{2 \pi} \delta\left(\omega_{1}+\ldots \omega_{p}\right) \mathcal{F}_{p}\left(\left\{\boldsymbol{k}_{j}, \omega_{j}\right\}\right)
$$

Here $\left\{\boldsymbol{k}_{j}, \omega_{j}\right\}$ and $\left\{\boldsymbol{k}_{j}\right\}$ are sets of corresponding variables with $j=1, \ldots p$. The transformation from $\boldsymbol{k}$ representation of $\boldsymbol{F}_{p}\left(\left\{\boldsymbol{k}_{j}\right\}\right)$ to the $p^{\text {th }}$-order structure function is done as follows: define the longitudinal component of the velocity as

$$
S_{p}(r)=\left\langle\left\{\left[\boldsymbol{u}\left(\frac{\boldsymbol{r}}{2}\right)-\boldsymbol{u}\left(-\frac{\boldsymbol{r}}{2}\right)\right] \cdot \frac{\boldsymbol{r}}{r}\right\}^{p}\right\rangle .
$$

Each of the factors is Fourier transformed according to

$$
\begin{array}{r}
\quad\left[\boldsymbol{u}\left(\frac{\boldsymbol{r}}{2}\right)-\boldsymbol{u}\left(-\frac{\boldsymbol{r}}{2}\right)\right]=\int \frac{d \boldsymbol{k}_{j}}{(2 \pi)^{3}} \hat{\boldsymbol{u}}\left(\boldsymbol{k}_{j}\right) \\
\times\left[\exp \left(i \frac{\boldsymbol{k}_{j} \cdot \boldsymbol{r}}{2}\right)-\exp \left(-i \frac{\boldsymbol{k}_{j} \cdot \boldsymbol{r}}{2}\right)\right],
\end{array}
$$

Accordingly,

$$
\begin{aligned}
S_{p}(r) & =(2 \pi)^{3} \int_{-\infty}^{\infty} \prod_{i=1}^{p} \frac{d \boldsymbol{k}_{i}}{(2 \pi)^{3}} \delta\left(\boldsymbol{k}_{1}+\ldots \boldsymbol{k}_{p}\right) \\
& \times f_{p}\left(\boldsymbol{r},\left\{\boldsymbol{k}_{j}\right\}\right) F_{p}\left(\left\{\boldsymbol{k}_{j}\right\}\right) .
\end{aligned}
$$

Here

$$
(2 \pi)^{3} F_{p}\left(\left\{\boldsymbol{k}_{j}\right\}\right) \delta\left(\boldsymbol{k}_{1}+\ldots \boldsymbol{k}_{p}\right)=\left\langle\prod_{j=1}^{p} \hat{\boldsymbol{u}}\left(\boldsymbol{k}_{j}\right) \cdot \frac{\boldsymbol{r}}{r}\right\rangle .
$$

The functions $f_{p}\left(\boldsymbol{r},\left\{\boldsymbol{k}_{j}\right\}\right)$ are seen from Eq. (C4) to be:

$$
f_{p}\left(\boldsymbol{r},\left\{\boldsymbol{k}_{j}\right\}\right)=\prod_{j=1}^{p}\left[2 i \sin \left(\frac{1}{2} \boldsymbol{k}_{j} \cdot \boldsymbol{r}\right)\right]
$$

In the limit $r \rightarrow 0$

$$
f_{p}\left(\boldsymbol{r},\left\{\boldsymbol{k}_{j}\right\}\right) \propto \prod_{j=1}^{p}\left(\boldsymbol{k}_{j} \cdot \boldsymbol{r}\right)
$$

The K41 scaling exponents $y_{p}$ associated with $p^{\text {th }}$-order correlation function $\mathcal{F}_{p}\left(\left\{\boldsymbol{k}_{j}, \omega_{j}\right\}\right) \propto k^{-y_{p}}$ in $(\boldsymbol{k}, \omega)$ representation is 


$$
y_{p}=4 p-11 / 3 \text {. }
$$

This corresponds to $\boldsymbol{S}_{p}(\boldsymbol{r}) \propto r^{p / 3}$ under the condition of convergence of integrals (C1, C5).

Next consider the 3rd order Green's function, $G_{3,3}\left(\left\{k_{j}, \kappa_{j}\right\}\right)$ in which we denoted by $k_{j}$ the set of incoming wave vectors and by $\kappa_{j}$ the set of outgoing wave vectors. The skeleton diagram of $G_{3,3}^{\mathrm{s}}\left(\left\{k_{j}, \kappa_{j}\right\}\right)$ which involves 4-point rungs is shown as diagram (3) in Fig. Aa. (The contribution of 6-point rungs to the skeleton is considered in Appendix $\mathrm{G}$ and shown not to change the present considerations). This skeleton has two rungs, and we consider it in the limit that the incoming $k_{j}$ vectors are much larger than the outgoing $\kappa_{j}$. In this limit we have four Green's functions with large $k$, contributing $\gamma_{k}^{-4}$, and one vertex with all $k$ large, contributing $k$. The two rungs have large $k$ vector in them [ $k_{5}$ in Eq. (22)], giving $k^{6}$. Finally, one of the rungs has large $k$ coming and going, and Eq. (22) requires for it a $k^{2 / 3}$. Altogether this gives $G_{3,3}\left(\left\{k_{j}, \kappa_{j}\right\}\right) \propto k^{x_{3}}$ with $x_{3}=25 / 3$ which is equal to $y_{3}$ given by Eq. C9). This means that the skeleton diagrams for $G_{3,3}\left(\left\{k_{j}, \kappa_{j}\right\}\right)$ (with asymptotics of the rung defined by the two-point fusion rules) automatically reproduces the K41 scaling exponent $\zeta_{3}=1$ in the three-point fusion. This is true subject to the condition that the integrals $(\mathrm{C} 1, \mathrm{C} 5)$ for $p=3$ converge. That this is so may be shown by a direct calculation. For future purposes it is extremely important to note that the principal contribution to the $\boldsymbol{k}$ integral (C5) comes from the region where $k_{1} \sim k_{2} \sim k_{3} \sim 1 / r$.

Now let us compare diagram (3) in Fig. 1 a and Fig. 5 with the skeleton diagrams for $G_{3,3}$ and $G_{4,4}$. One recognizes that in general for $G_{p, p}$, we will have $(p-1)$ rungs with large incoming $k$, contributing $k^{-3(p-1)}$ [originating from $k_{e}$ in Eq. (22)]. We will have also $2 p-2$ Green's functions with large $k$ contributing $k^{-(2 p-2) 2 / 3}$. Next we will have $p-2$ outgoing legs with large $k$ contributing $k^{-(p-2) 2 / 3}$ from Eq. (9). Finally we will have $2 p-2$ vertices having incoming and outgoing large $k$ vectors, contributing $k^{p-2}$. All together we find that $G_{p, p}\left(\left\{k_{j}, \kappa_{j}\right\}\right) \propto k^{-x_{p}}$ with $x_{p}=4 p-11 / 3$ which is equal to $y_{p}$ given by Eq. (C9). Convergence of the $\boldsymbol{k}$ integral (C5) for $p=4$ may be shown by direct calculations. A proof of convergence of the $\boldsymbol{k}$ integrals (C5) for $p>4$ is a tedious exercise which nevertheless may be done, for example, iteratively. It is readily demonstrated that the integral converges when all $k_{j}$-vectors are of the same order of magnitude (say, $k$ ). Then $G_{p, p} \sim k^{11 / 3-4 p}$. After $(p-1) \omega$-integrations (each of them giving a factor $k^{2 / 3}$ ) one has $k^{3(p-1)-p / 3}$ which is enough for convergence of $(p-1) d^{3} k$ integrals in the UV region $k_{j} \sim k \gg 1 / r$. In the IR region $k_{j} \sim k \ll 1 / r$ the functions $f_{p}$ provide the integral with additional $k^{p}$ factor [according to (C8)] which guarantees the convergence.

The considerations of the 6-point and higher order rungs leave these conclusions invariant.

\section{APPENDIX D: ANALYSIS OF TWO LOOP INTEGRALS CONTRIBUTING TO $\zeta_{2}$}

The integrand in the integral (66) is a function of $q_{1}$ and $q_{2}$ and it depends on $k$ and $\kappa$ as parameters. The integration range is the $q_{1}-q_{2}$ infinite plane, but in the limit $k \gg \kappa$ the main contribution comes form the four finite quadrants $\kappa<\left|q_{1}\right|,\left|q_{2}\right|<k$. Well inside the quadrants we are allowed to use the asymptotic form in which $\kappa \ll\left|q_{1}\right|,\left|q_{2}\right| \ll k$. In this regime the integrand is $k, \kappa$ independent, and the dependence of the integrals on $k, \kappa$ appears only via the limits of integration. By changing the dummy variables $q_{1}$ and $q_{2}$ we can now project all four quadrants into one of them, say $q_{1}$ and $q_{2}$ positive. In this asymptotic regime we can use for the rungs in the integrand of (56) that include either $k$ or $\kappa$ their asymptotic form (32). This results in

$$
\begin{aligned}
K(k, \kappa) & =\tilde{\delta}^{2} \int_{p}^{k} \frac{d q_{1}}{q_{1}} \int_{p}^{k} \frac{d q_{2}}{q_{2}} \Psi\left(q_{1}, q_{2}\right), \\
\Psi\left(q_{1}, q_{2}\right) & =\tilde{\Psi}\left(q_{1}, q_{2}\right)-\tilde{\Psi}\left(-q_{1}, q_{2}\right) .
\end{aligned}
$$

In Appendix $\mathrm{E}$ we show how to analyze this kind of integral with the aim of extracting the coefficients of the leading and first subleading logarithmic terms, i.e.

$$
K_{1}(k, \kappa)=\frac{a_{1}}{2} \ln ^{2}(k / \kappa)+b_{1} \ln (k / \kappa)
$$

Using the results there (E4) with $\tilde{\Psi}\left(q_{1}, q_{2}\right)=$ $\tilde{\Psi}_{1}\left(q_{1}, q_{2}\right)$,

$$
\tilde{\Psi}_{1}\left(q_{1}, q_{2}\right)=\frac{q_{1}^{3}\left|q_{1}-q_{2}\right| \operatorname{sign}\left(q_{2}\right)}{2\left(q_{1}^{2}-q_{1} q_{2}+q_{2}^{2}\right)^{2}},
$$

one find immediately $a \rightarrow a_{1}=1$ as required by the anticipated expansion employed in Eqs. (44)-(46). To compute $b_{1}$ we examine the integral $b_{1}(A)$ numerically, see Fig. 7. We see that the requested limit exists and that $b_{1} \approx-0.434$.

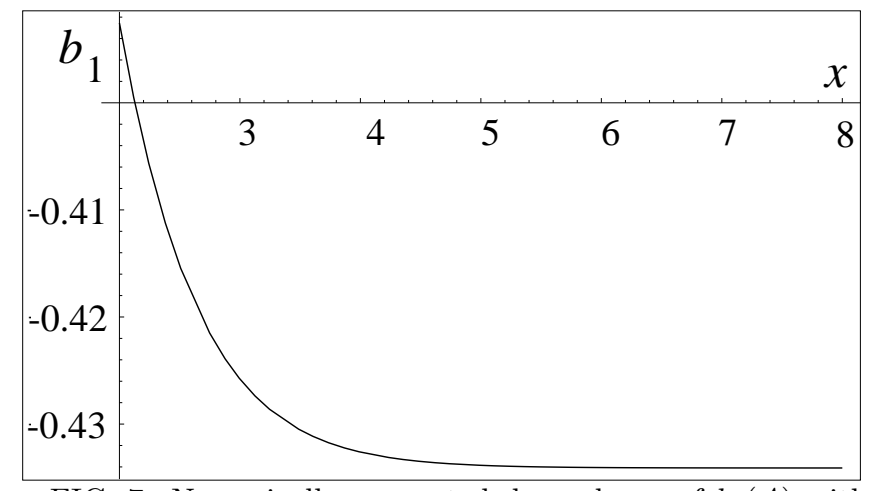

FIG. 7. Numerically computed dependence of $b_{1}(A)$ with $A=\exp (x)$. 


\section{APPENDIX E: EXTRACTION OF THE SUBLEADING LOGARITHMIC TERM FROM THE 2-LOOP INTEGRALS}

The 2-loop integrals have the characteristic structure appearing in (D1)

$$
I(A)=\tilde{\delta}^{2} \int_{1}^{A} \frac{d q_{1}}{q_{1}} \int_{1}^{A} \frac{d q_{2}}{q_{2}} \Psi\left(q_{1}, q_{2}\right),
$$

where $A \gg 1$ and $\Psi\left(q_{1}, q_{2}\right)$ is homogeneous function of degree zero: $\Psi\left(\lambda q_{1}, \lambda q_{2}\right)=\Psi\left(q_{1}, q_{2}\right)$. When $\Psi\left(q_{1}, q_{2}\right)=$ 1 then $I(A)=\ln ^{2} A$. In general only the leading term of $I(A)$ is proportional to $\ln ^{2} A$ and we expect the following subleading terms:

$$
I(A)=\tilde{\delta}^{2}\left[\frac{a}{2} \ln ^{2} A+b \ln A+c+\frac{d}{A}+\ldots\right]
$$

Our goal is to find the coefficient $b$ in the limit $A \rightarrow \infty$. Taking the first derivative of (E2) with respect to $A$ and multiplying by $A$ we find

$$
\begin{aligned}
& a \ln A+b-\frac{d}{A}-\ldots=\int_{1}^{A} \frac{d q_{2}}{q_{2}} \Psi\left(1, q_{2}\right)+\int_{1}^{A} \frac{d q_{1}}{q_{1}} \Psi\left(q_{1}, 1\right) \\
& =\int_{1 / A}^{1} \frac{d x}{x} \Psi(x, 1)+\int_{1 / A}^{1} \frac{d y}{y} \Psi(1, y)
\end{aligned}
$$

where we changed the dummy variables $q_{1}=x A$ and $q_{2}=y A$. Taking another derivative and multiplying by $A$ we find for large $A$

$$
a=\Psi\left(1, \frac{1}{A}\right)+\Psi\left(\frac{1}{A}, 1\right) .
$$

Substituting this result in (E3), and representing $\ln A$ as $\int_{1 / A}^{1} d x / x$ we find

$$
\begin{aligned}
b & =\lim _{A \rightarrow \infty} b(A), \\
b(A) & =\int_{1 / A}^{1} \frac{d x}{x}\left[\Psi(x, 1)+\Psi(1, x)-\Psi\left(\frac{1}{A}, 1\right)-\Psi\left(1, \frac{1}{A}\right)\right] .
\end{aligned}
$$

If the expansion assumed in Eq. (E2) is valid, this limit must exist.

\section{APPENDIX F: THE NINE 2-LOOP DIAGRAMS OF $G_{3,3}$}

Consider diagram (1a) in Fig. 8. We are interested in the ratio of $T_{3,1 a}^{(2)} / T_{3}^{\mathrm{s}}$, where $T_{3,1 a}^{(2)}$ is obtained by substituting the diagram (1a) instead of $G_{3,3}$ in Eq. (49). In the asymptotic regime $\kappa r \ll 1$ the loop integrals over $q_{1}$ and $q_{2}$ contribute mostly in the regime $k \gg q_{1}, q_{2} \gg \kappa$. In this regime the integrals over $k_{a}, \omega_{a}, k_{b}, \omega_{b}$ cancel in the desired ratio. Similarly the Green's functions $G\left(\kappa_{d}\right)$, $G\left(\kappa_{e}\right)$ and $G\left(\kappa_{f}\right)$ also cancel in the ratio. Accordingly
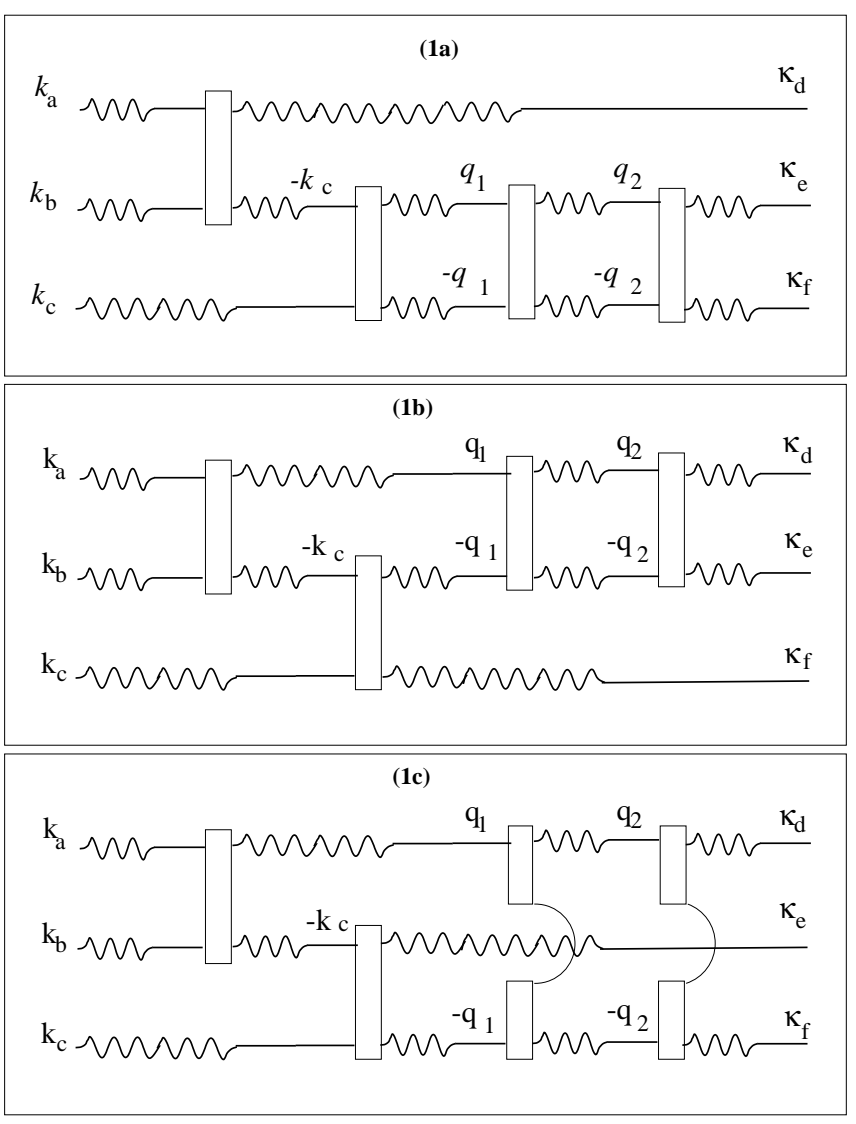

FIG. 8. The first group of three 2-loop diagrams appearing in the loop expansion of $G_{3,3}$.

$T_{3,1 a}^{(2)} / T_{3}^{\mathrm{s}}$ can be calculated from the amputated diagram (2) in Fig. 10, in which the explicit dependence on $k_{j}$ and $\kappa_{j}$ has disappeared. These wavevectors remain only in the limits of the integrals over $q_{1}$ and $q_{2}$, with $k$ replaced by $1 / r$. In this diagram every black dot contributes a factor of $q_{j}^{1 / 3+\delta_{a}}$ where $q_{j}$ is the wavevector on the right of the black dot. This is a remnant of the corresponding rung before the amputation. The thin line connecting these dots is just a reminder that we have loop integrals to perform.

The point to understand now is that if we use diagrams (1b) and (1c) in Fig. 8 to form $T_{3,1 b}^{(2)}$ and $T_{3,1 c}^{(2)}$, the ratio of these to $T_{3}^{\mathrm{s}}$ can be again calculated from the amputation of their own diagrams. This will lead to the identical amputated diagram (2) of Fig. 10. In addition, and most importantly, the integral that needs to be computed is the same as Eq. (66). Thus one recaptures Eq. (67) but with the combinatorial factor 3 in front of the RHS:

$$
\begin{aligned}
T_{3,1 a+1 b+1 c}^{(2)}(r, \kappa) & =3 \tilde{\delta}^{2}\left[\frac{1}{2} \ln ^{2}\left(\frac{1}{\kappa r}\right)\right. \\
& \left.+b_{1} \ln \left(\frac{1}{\kappa r}\right)\right] T_{3}^{\mathrm{s}}(r, \kappa),
\end{aligned}
$$

with $b_{1}$ of Eq. (68). 


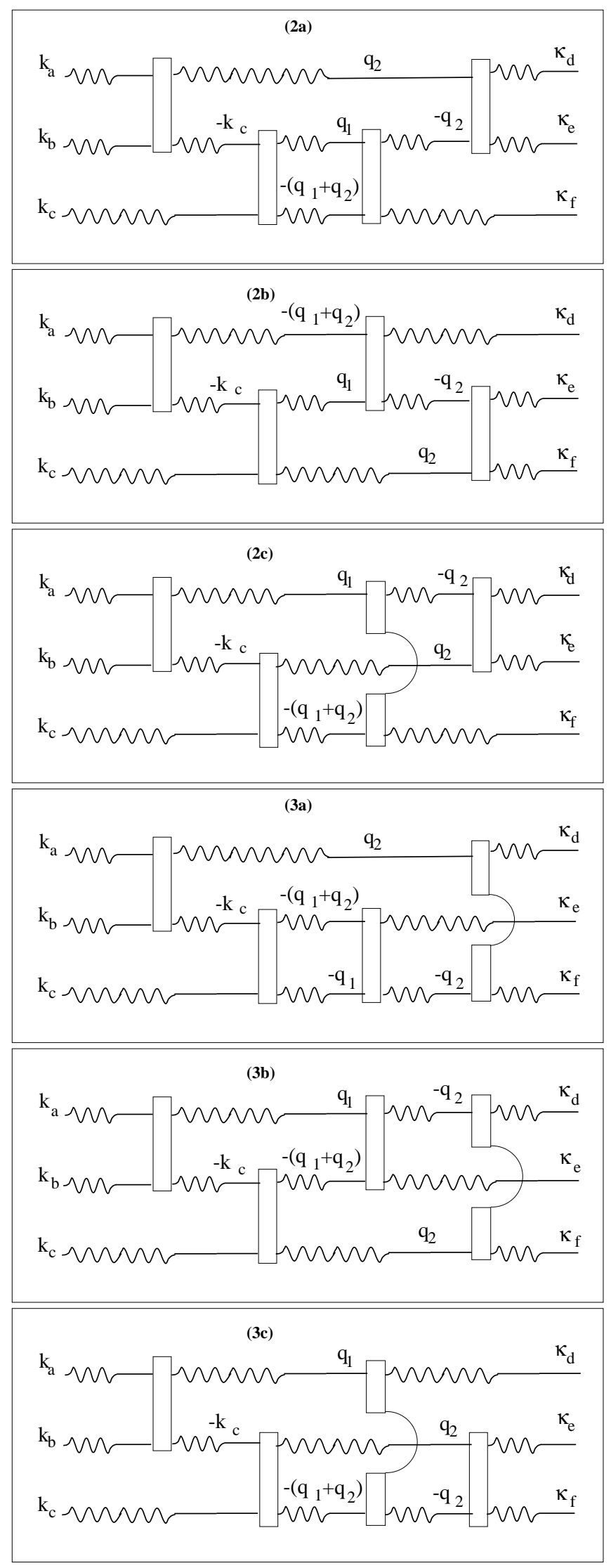

FIG. 9. The second group of six 2-loop diagrams appearing in the loop expansion of $G_{3,3}$.

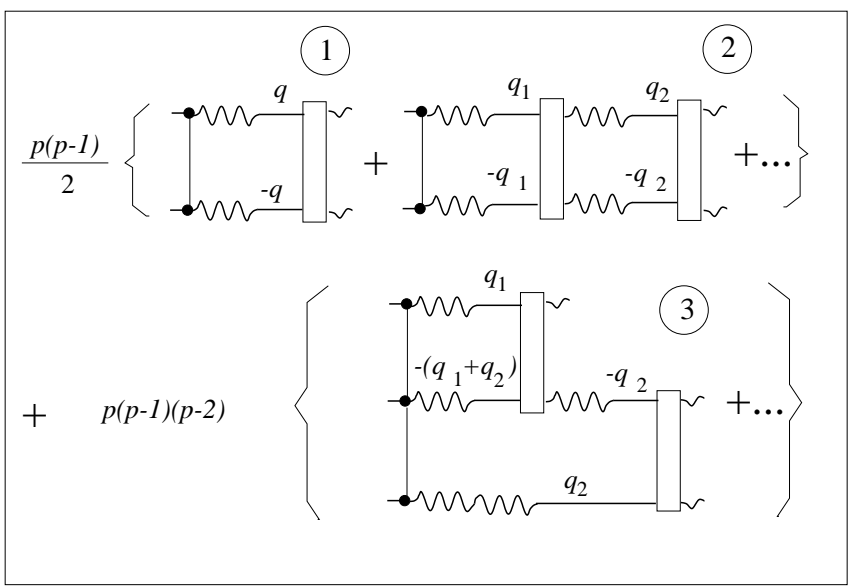

FIG. 10. The amputated diagrams that appear up to 2-loop in the loop expansion of $G_{p, p}$, with the appropriate combinatorial factors.

The second group of six diagrams $(2 \mathrm{a}-3 \mathrm{c})$ shown in Fig. 9 yields to a similar analysis, but the amputated diagram is shown as diagram (3) in Fig. 10. All six diagrams result in the very same amputation, up to permutations of the three struts. Analyzing the amputated diagram (3) one brings it to the canonical form (D1) with $\tilde{\Psi}\left(q_{1}, q-2\right)$ given by (70). Accordingly we write

$T_{3,2 a, \ldots 3 c}^{(2)}(r, \kappa)=6 \tilde{\delta}^{2}\left[\frac{1}{2} \ln ^{2}\left(\frac{1}{\kappa r}\right)+b_{2} \ln \left(\frac{1}{\kappa r}\right)\right] T_{3}^{\mathrm{s}}(r, \kappa)$,

with $b_{2}$ of (71).

The analysis of the 2-loop diagrams that involve 4point rungs in the context of $G_{p, p}$ follows exactly the same lines, with the amputated diagrams being those of Fig. 10. The only thing to mind is the combinatorics, which are presented explicitly in Fig. 10, leading to the numbers in Eq. (75).

\section{APPENDIX G: RESUMMED EQUATIONS FOR THE 4-POINT AND 6-POINT RUNGS}

In this Appendix we sketch a theory for the 4-point and 6-point rungs. Our main aim here is to explain why the 6-point rung is quadratic in the smallness, but we use the opportunity to indicate how a future theory of these objects may be formulated.

Consider the beginning of the series expansion of the 4-point rung which is shown in Fig. 2bb. Diagram (2) contains a cross of correlators each attached to two 3-point vertices. This is exactly diagram (1), and therefore the equation can lend itself to resummation resulting in the equation shown in Fig. 11a. We note that this is not the full equation for the 4-point rung even in 1-loop order 

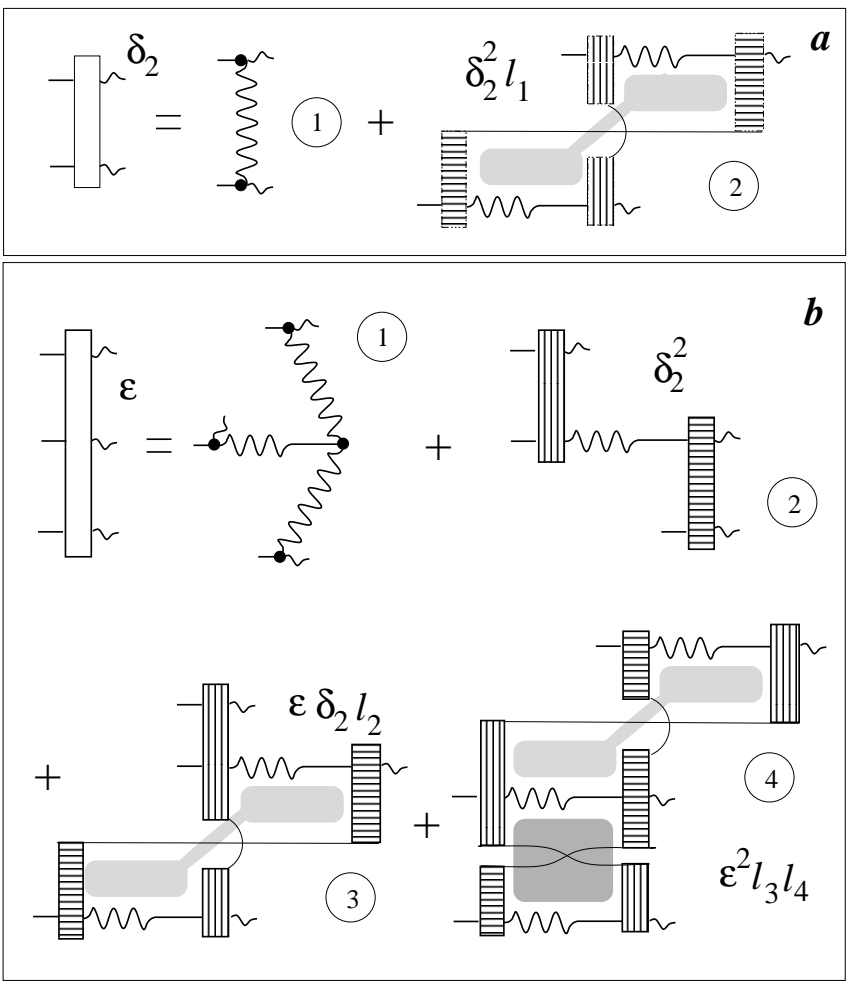

FIG. 11. A sketch of the equations for the 4-point rung (panel a) and 6-point rung (panel b). The shading of rungs is used to identify different pieces of the same object. In the same manner we shade the loops to identify one or two loop integrals.

since we did not take into account the ladders with a correlator and Green's function in a cross section. Taking into account all the needed contributions is not difficult, but is not the main point of this Appendix, and we proceed for simplicity without the additional terms.

In the asymptotic regime the bare contribution diagram (1) in Fig. 11a is negligible. With this contribution discarded, the remaining equation is homogeneous, calling for finding a zero mode of the equation. Since we have already demonstrated that the 4-point rung is small, of the order of $\delta_{2}$ we can conclude that the loop integral which we denote as $\ell_{1}$ must be large, or the order of $1 / \delta_{2}$. (the homogeneous equation can be only solved if $\left.\delta_{2} \approx \delta_{2}^{2} \ell_{1}\right)$. In fact, in the future it would be extremely worthwhile to solve the full equation in the 1-loop order and demonstrate that this is the case, and thus to lend further weight to the theory presented in this paper. Of course solving such an equation will also supply us with a functional form of the 4-point rung, and with it a substantial part of the value of the parameter $b_{2}$ which appears in the final result for the scaling exponents.

In Fig. 11 b we present the resummed form of the equation of the 6-point rung, to the same level of qualitative discussion. Again we discard in the asymptotic limit the bare contribution of diagram (1), but we cannot neglect diagram (2) since it has the same asymptotic behavior as the resummed 6-point rung. Diagram (2) is of the order of $\delta_{2}^{2}$. Diagram (3) is of the order of $\epsilon \delta_{2} \ell_{2}$ where $\ell_{2}$ is the loop integral. This integral is very similar to $\ell_{1}$, and we therefore estimate $\ell_{2} \approx \ell_{1} \approx 1 / \delta_{2}$, and thus diagram (3) is of the order of the LHS. Diagram (4) is of the order of $\epsilon^{2} \ell_{3} \ell_{4}$ where $\ell_{3}$ and $\ell_{4}$ each refers to one of the loop integrals. With the same level of approximation we estimate it thus to be of $O\left(\epsilon^{2} / \delta_{2}^{2}\right)$. Denoting $x \equiv \epsilon / \delta^{2}$ we thus represent the order of magnitude relations that result from panel $b$ by the equation

$$
x=1+a x+b x^{2},
$$

where $a$ and $b$ are dimensionless constants of $O(1)$. It is obvious that only $x \approx 1$ is a consistent solution of this equation, and we thus conclude that the 6-point rung is quadratic in the smallness $\delta_{2}$.

We therefore understand that the 6-point rung appears in our considerations only at the level of the $O\left(\delta^{2}\right)$ order. In this order it appears in addition to the 2-loops integrals which are formed by two 4-point rungs, as discussed in detail in the text of the paper. But since the 6-point rung connects three struts, exactly like the structure made of two 4-point rungs, the combinatorical factors appearing in the $p$ th order scaling exponents are identically the same. Accordingly we understand that the effect of the 6 -point rung is only in renormalizing the value of the parameter $b_{2}$ which anyway is model dependent.

Similar consideration apply to the 8-point rung which begins to affect the theory only in $O\left(\delta_{2}^{3}\right)$. It will renormalize the value of the parameter $b_{3}$ in Eq. $(78)$. Higher order rungs are even less relevant for the calculation at hand.

[1] A.N. Kolmogorov, Dokl. Acad. Nauk SSSR 30, 9 (1941).

[2] A. S. Monin and A. M. Yaglom. Statistical Fluid Mechanics: Mechanics of Turbulence, volume II. (MIT Press, Cambridge, Mass., 1973).

[3] F. Anselmet, Y. Gagne, E.J. Hopfinger, and R.A. Antonia, J. Fluid Mech. 140, 63 (1984).

[4] K.R. Sreenivasan and P. Kailasnath. Phys. Fluids A 5 512, (1993).

[5] R. Benzi, S. Ciliberto, R. Tripiccione, C. Baudet, F. Massaioli and S. Succi, Phys. Rev. E 48, R29 (1993).

[6] Uriel Frisch. Turbulence: The Legacy of A.N. Kolmogorov. (Cambridge University Press, Cambridge, 1995).

[7] A. N. Kolmogorov, J. Fluid Mech. 13, 82 (1962).

[8] R.H. Kraichnan, Phys.Fluids 11, 945 (1968).

[9] R.H. Kraichnan, Phys. Rev. Lett. 72, 1016 (1994).

[10] K. Gawedzki and A. Kupiainen, Phys. Rev. Lett. 75, 3608 (1995)

[11] I. Arad, V. S. L'vov, E. Podivilov and I. Procaccia, "Anomalous Scaling in the Anisotropic Sectors of the 
Kraichnan Model of Passive Scalar Advection", Phys. Rev. E, in press. Also: \# chao-dyn/9907017

[12] L. Ts. Adzhemyan, N.V. Antonov and A.V. Vasil'ev Phys. Rev. E 58, 1823 (1998).

[13] Y. Cohen, V.S. L'vov, A. Pomyalov and I. Procaccia "A Simple, Perturbative Calculation of the Anomalous Scaling Exponents in the Kraichnan Model of Passive Scalar Advection", in preparation.

[14] V.I. Belinicher, V.S. L'vov, A.Pomyalov and I. Procaccia, J. of Stat. Phys. 93, 797(1998)

[15] V.S. L'vov and I. Procaccia, Phys. Rev. E 52, 3858 (1995).

[16] V.S. L'vov and I. Procaccia, Phys. Rev. Lett. 76, 2896 (1996).

[17] V.S. L'vov and I. Procaccia, Phys. Rev. E 53, 3468 (1996).

[18] V.S. L'vov and I. Procaccia, Phys. Rev. E, 52, 3840
(1995)

[19] H. W.Wyld, Ann. Phys. 14, 143 (1961).

[20] P.C. Martin, E.D. Siggia, H.A. Rose, Phys. Rev. A 8, 423 (1973).

[21] V.S. L'vov and I. Procaccia "Exact Resummation in the Theory of Hydrodynamic Turbulence": 0. Line Resummed Diagrammatic Perturbation Approach" in F. David P. Ginsparg, and J. Zinn-Justin, eds. Les Houches session LXII, 1994, "Fluctuating Geometries in Statistical Mechanics and Field Theory" (Elsevier, 1995).

[22] V.I. Belinicher and V.S. L'vov Zh. Eksp. Teor. Fiz. 93, 1269 (1987), [Soviet Physics - JETP. 66, 303 (1987)].

[23] R.H. Kraichnan, J. Fluid Mech. 83, 349 (1977).

[24] V.S. L'vov and V. Lebedev. Phys. Rev.E, 471794 (1993).

[25] V.S. L'vov, E. Podivilov and I. Procaccia. Phys. Rev. E 55, 7030 (1997). 